\title{
Oral ferroportin inhibitor ameliorates ineffective erythropoiesis in a model of $\boldsymbol{\beta}$-thalassemia
}

\author{
Vania Manolova, Naja Nyffenegger, Anna Flace, Patrick Altermatt, Ahmet Varol, Cédric Doucerain, \\ Hanna Sundstrom, and Franz Dürrenberger \\ Vifor (International) Ltd., St. Gallen, Switzerland.
}

\begin{abstract}
$\beta$-Thalassemia is a genetic anemia caused by partial or complete loss of $\beta$-globin synthesis, leading to ineffective erythropoiesis and RBCs with a short life span. Currently, there is no efficacious oral medication modifying anemia for patients with $\boldsymbol{\beta}$-thalassemia. The inappropriately low levels of the iron regulatory hormone hepcidin enable excessive iron absorption by ferroportin, the unique cellular iron exporter in mammals, leading to organ iron overload and associated morbidities. Correction of unbalanced iron absorption and recycling by induction of hepcidin synthesis or treatment with hepcidin mimetics ameliorates $\beta$-thalassemia. However, hepcidin modulation or replacement strategies currently in clinical development all require parenteral drug administration. We identified oral ferroportin inhibitors by screening a library of small molecular weight compounds for modulators of ferroportin internalization. Restricting iron availability by VIT-2763, the first clinical stage oral ferroportin inhibitor, ameliorated anemia and the dysregulated iron homeostasis in the $\mathrm{Hbb}^{\text {th } 3 /+}$ mouse model of $\beta$-thalassemia intermedia. VIT-2763 not only improved erythropoiesis but also corrected the proportions of myeloid precursors in spleens of $\mathrm{Hbb}^{\text {th } 3 /+}$ mice. VIT-2763 is currently being developed as an oral drug targeting ferroportin for the treatment of $\beta$-thalassemia.
\end{abstract}

\section{Introduction}

$\beta$-Thalassemia is an inherited anemia caused by mutations in the $\beta$-globin gene of hemoglobin $(\mathrm{Hb})$ resulting in abnormal RBCs with decreased life span $(1,2)$. In healthy subjects, $\mathrm{Hb}$ is composed of $\alpha$ - and $\beta$-globin chains, which, together with the iron-containing heme groups, form functional $\alpha_{2} \beta_{2}$ heterotetramers within RBCs to efficiently deliver oxygen to tissues. The main pathophysiological mechanism in $\beta$-thalassemia results from the decreased synthesis of $\beta$-globin chains, causing accumulation of unpaired $\alpha$-globin aggregates on the membranes of RBCs. Precipitated $\alpha$-globin aggregates contain heme and iron, which generate ROS leading to a shortened life span of RBCs, anemia, and tissue hypoxia $(3,4)$. As a compensatory response to the reduced life span of RBCs in $\beta$-thalassemia patients, erythropoiesis is greatly stimulated, leading to increased proliferation and decreased differentiation of erythroid precursors (ineffective erythropoiesis) in BM and extramedullary sites, such as spleen and liver (5). The ineffective erythropoiesis in $\beta$-thalassemia substantially contributes to the anemia and causes iron overabsorption to support the increased iron demand for $\mathrm{Hb}$ synthesis, leading to organ iron overload. Liver, spleen, heart, and pancreas are the tissues commonly affected by iron

Conflict of interest: All authors are employees of Vifor (International) Ltd. and might own equities. VM and FD are inventors in patents related to the publication (W02017/068089 and W02017/068090).

Copyright: (5) 2020, American Society for Clinical Investigation.

Submitted: April 11, 2019; Accepted: October 8, 2019; Published: December 9, 2019.

Reference information: J Clin Invest. 2020;130(1):491-506.

https://doi.org/10.1172/JCl129382. overload, and without therapeutic intervention, iron overload may lead to organ damage, such as liver cirrhosis, heart failure, and diabetes.

The homeostatic regulation of iron absorption and distribution is controlled by hepcidin, a 25 aa peptide produced mainly in the liver (6). Hepcidin inhibits ferroportin (SLC4OA1), the only known cellular iron exporter $(7,8)$. Ferroportin on the basolateral membrane of intestinal enterocytes transfers dietary iron into the plasma; on spleen and liver macrophages, it exports recycled iron from the $\mathrm{Hb}$ of senescent $\mathrm{RBCs}$, and ferroportin on hepatocytes exports iron from liver stores (9). Recently, Zhang et al. have demonstrated that ferroportin is highly abundant on mature RBCs and has an essential role in maintaining systemic iron homeostasis and protecting RBCs from oxidative stress $(10,11)$. Hepcidin binds to and triggers ferroportin internalization and degradation, which causes a rapid drop in blood iron levels (12). In addition, hepcidin inhibits iron export, not only by causing ferroportin endocytosis, but also by directly occluding the transporter (13). Hepcidin transcription is downregulated by erythropoiesis, iron deficiency, and hypoxia, whereas inflammation and high systemic iron levels lead to hepcidin upregulation $(6,14)$.

The ineffective erythropoiesis in $\beta$-thalassemia causes iron overabsorption due to feedback compensatory response to hypoxia, which suppresses hepcidin (15). Since the abnormally high iron levels that result from the suppression of hepcidin further stimulate erythropoiesis, anemia and iron overload are worsened in a vicious circle. HIF $2 \alpha$ plays a major role in linking erythropoiesis and iron absorption, as HIF2 $\alpha$ stimulates both erythropoietin (EPO) production and upregulates expression of the divalent metal transporter 1 (DMT1), duodenal cytochrome B (DCytB), and ferroportin $(14,16)$. EPO 
A

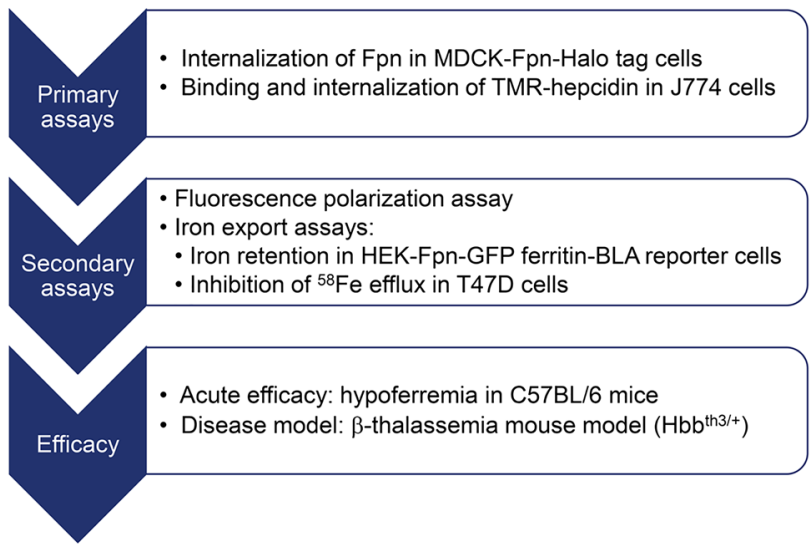

triggers the proliferation of erythroblasts and induces the production of the erythroid factor erythroferrone (ERFE), which in turn suppresses hepcidin (17).

Therefore, correction of unbalanced iron absorption by induction of hepcidin synthesis or supplementation of hepcidin mimetics is an attractive therapeutic approach for normalizing the dysregulated iron metabolism in $\beta$-thalassemia. Experimental drugs, such as shortened hepcidin-derived peptides containing unnatural amino acids (minihepcidins) or oligonucleotides that increase endogenous hepcidin synthesis by inhibition of Tmprss6, have been shown to correct anemia and decrease iron overload in the $\mathrm{Hbb}^{\mathrm{th} / \mathrm{t}}$ mouse model of $\beta$-thalassemia intermedia (18-20). In addition, synthetic human hepcidin (LJPC-401) as well as a hepcidin peptidomimetic (PTG-300) and an anti-sense oligonucleotide targeting Tmprss6 (IONIS-TMPRSS6- $\mathrm{L}_{\mathrm{RX}}$ ) have been tested in phase I clinical studies. Hepcidin modulation or replacement strategies currently in clinical development all require parenteral administration. Orally bioavailable minihepcidins have been shown to lower serum iron in WT mice (21). Nevertheless, presently no clinical data for an oral drug targeting ferroportin have been published. Oral drug administration offers advantages over parenteral, such as the ease of administration by patients, in particular children, high degree of flexibility on dosages and formulation, cost effectiveness, fewer sterility constraints, and no risk of injection site reactions and infection. Parenteral administration of drugs usually requires medical attendance, which further increases treatment costs and may negatively affect patient compliance.

The scope of the present publication is to describe the profile and mode of action of the compound VIT-2763, an oral small molecule inhibitor of ferroportin. Based on the promising preclinical efficacy and tolerability profile, VIT-2763 has entered clinical development (22). Since no oral ferroportin inhibitors or hepcidinmimetic drugs are available for the treatment of iron overload and ineffective erythropoiesis, VIT-2763 is considered the first oral drug candidate to reach the clinical development stage.

\section{Results}

Ferroportin inhibitors were discovered by screening a small molecule library. Ferroportin inhibitors were identified by screening a library of small molecular weight compounds (250,000 compounds) for modulators of ferroportin internalization using Madin-Darby canine kidney (MDCK) cells expressing fluorescently tagged human ferroportin. Confirmed hit compounds were then tested for
B

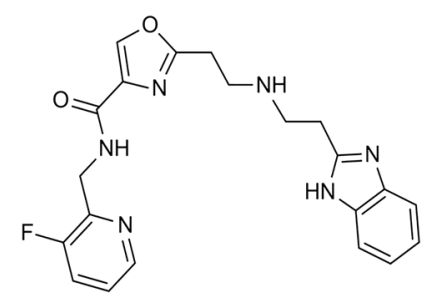

Figure 1. Identification of ferroportin inhibitors. (A) Screening and profiling cascade used to identify ferroportin inhibitors. (B) Chemical structure of the ferroportin inhibitor VIT-2763. their ability to inhibit binding and internalization of fluorescently labeled hepcidin (6-carboxytetramethylrhodamine hepcidin [TMR-hepcidin]) in the mouse macrophage cell line J774, which expresses endogenous ferroportin. In addition, a fluorescence polarization binding assay was used to more directly demonstrate inhibition of TMR-hepcidin binding to purified recombinant human ferroportin. Compounds that showed inhibition of TMR-hepcidin binding to ferroportin were further profiled with functional assays, including ferroportin internalization and iron efflux assays (Figure 1A). Lead structures were optimized for potency, drug metabolism, and pharmacokinetics (PKs) parameters by medicinal chemistry, and selected compounds were tested for acute efficacy in inducing hypoferremia in C57BL/6 mice. Finally, a small number of preclinical candidates were tested for efficacy in the $\mathrm{Hbb}^{\mathrm{th} 3 /+}$ mouse model of $\beta$-thalassemia intermedia (Figure 1A).

The clinical compound, VIT-2763 (Figure 1B) is a small organic heterocyclic molecule that has been evaluated in biological assays as a salt of the organic base (MW $408.43 \mathrm{~g} / \mathrm{mol}$ ).

VIT-2763 inhibits hepcidin binding to ferroportin and blocks iron efflux. Potencies of ferroportin binding were compared between VIT-2763 and hepcidin in a competition assay using the macrophage cell line J774, in which expression of ferroportin can be triggered with iron. The small molecule VIT-2763 competed for binding and internalization of fluorescently labeled TMR-hepcidin with $\mathrm{IC}_{50}$ of $9 \pm 5 \mathrm{nM}$, mean $\pm \mathrm{SD}$, which was within the range of the potency of unlabeled synthetic hepcidin $\left(\mathrm{IC}_{50}=13 \pm 4 \mathrm{nM}\right.$, mean \pm $\mathrm{SD}$ ) in the same assay (Figure 2, A and B).

To investigate the potency of VIT-2763 in a cell-free assay without interference of ferroportin internalization, we used purified human ferroportin expressed in yeast in a fluorescence polarization assay. The binding of TMR-hepcidin to ferroportin leads to increased fluorescence polarization of the TMR-hepcidin ligand. Addition of VIT-2763 dose dependently reduced the fluorescence polarization signal, indicating that VIT-2763 displaces TMR-hepcidin from ferroportin (Figure 2C, $\mathrm{IC}_{50}$ of $24 \pm 13 \mathrm{nM}$, mean \pm SD). The binding of TMR-hepcidin to ferroportin was dose dependently inhibited by unlabeled full-length human hepcidin (hepcidin-25), although with a lower potency compared with VIT-2763 (Figure 2C, IC $_{50}$ of $533 \pm 250 \mathrm{nM}$, mean \pm SD). A truncated inactive version of hepcidin (hepcidin-20), lacking the first $5 \mathrm{~N}$-terminal amino acids involved in ferroportin binding (23), failed to decrease the fluorescence polarization signal (Sup- 


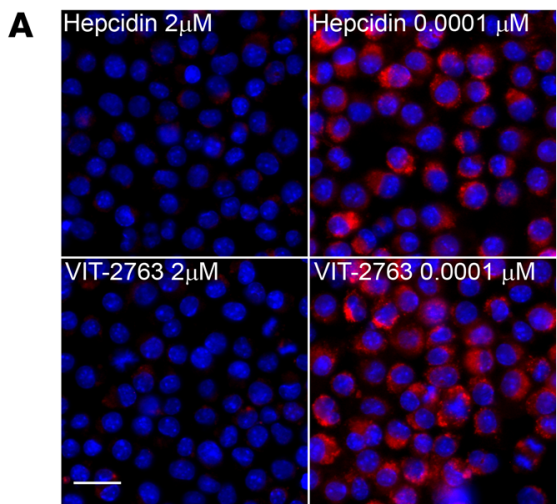

D

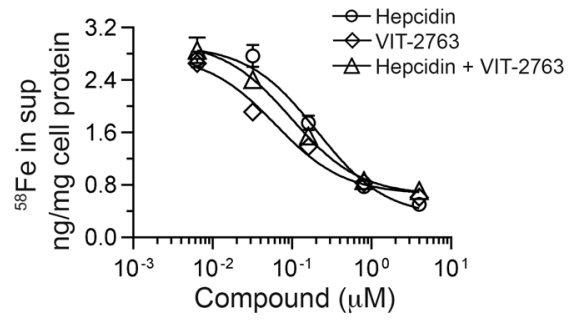

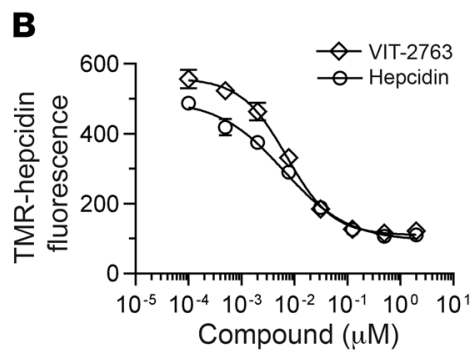


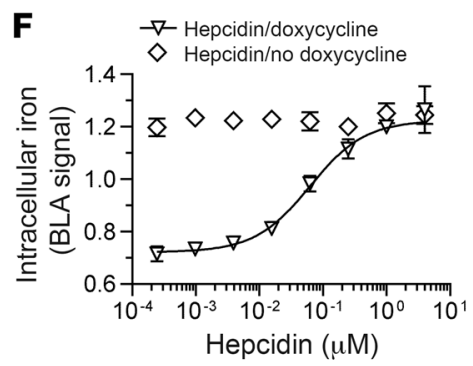

Figure 2. VIT-2763 competes with hepcidin for ferroportin binding. (A) VIT-2763 prevented the internalization of TMR-hepcidin in J774 cells. Representative fluorescence microscopy pictures from more than 10 independent experiments are shown with J774 cells at high $(2 \mu \mathrm{M})$ and low concentrations $(0.0001 \mu \mathrm{M})$ of VIT-2763 or hepcidin before adding TMR-hepcidin (red). Nuclei are shown in blue. Scale bar: $25 \mu \mathrm{m}$. (B) Dose-response curves of VIT-2763 and unlabeled hepcidin in 7774 TMR-hepcidin internalization assay. $n=3$ per concentration. (C) Dose-response curves of VIT-2763 and unlabeled hepcidin in fluorescence polarization assay. $n=3$ per concentration. (D) Dose-response curves in iron efflux assay in T47D cells. Shown are dose-response curves of VIT-2763 or hepcidin alone and both in a combination with equimolar concentrations. $n=3$ or 4 per concentration. (E and $\mathbf{F}$ ) Dose-response curves of VIT$2763(\mathbf{E})$ and hepcidin (F) in HEK-FPN1-GFP ferritin-BLA reporter assay with or without doxycycline induction of ferroportin. $n=4$ per concentration. Data are presented as mean + SD for each concentration.

plemental Figure 1; supplemental material available online with this article; https://doi.org/10.1172/JCI129382DS1). Furthermore, the potency of structurally related ferroportin inhibitors measured in J774 TMR-hepcidin internalization assay correlated significantly with the potency of the same compounds measured in the fluorescence polarization assay (Supplemental Figure 2). Based on these data, we concluded that the purified ferroportin shows ligand-binding properties similar to those of the native ferroportin expressed on the cell membrane.

The results with both TMR-hepcidin assays showed that VIT-2763 competes with hepcidin for ferroportin binding and internalization; however, these assays do not provide functional information for the effect of VIT-2763 on iron export activity of ferroportin. To address the effect of VIT-2763 on ferroportin function, the human breast cancer cell line T47D expressing endogenous ferroportin was incubated with iron sulfate labeled with the stable isotope ${ }^{58} \mathrm{Fe}$ for 20 hours and cells were treated with either VIT-2763 or hepcidin alone or both compounds added simultaneously. Hepcidin blocked the cellular iron efflux dose dependently, as measured by quantification of ${ }^{58} \mathrm{Fe}$ in the cell supernatant, with an average $\mathrm{EC}_{50}$ of $123 \pm 46$ $\mathrm{nM}$, mean \pm SD. Importantly, VIT-2763 inhibited the iron efflux with a slightly higher potency than hepcidin $\left(\mathrm{EC}_{50}\right.$ of $68 \pm 21 \mathrm{nM}$, mean $\pm \mathrm{SD}$ ). No additive effect was observed by preincubation (not shown) of either VIT-2763 or hepcidin or by coincubation of both at equimolar concentrations (Figure 2D).
To investigate whether the observed inhibition of cellular iron export by VIT-2763 is dependent on ferroportin, we used HEK293 cells expressing a doxycycline-inducible human ferroportin fused to GFP. In addition, this cell line contains the promoter and adjacent $5^{\prime}$ UTR with the iron regulatory element (IRE) of the human ferritin $\mathrm{H}$ gene fused to a $\beta$-lactamase (BLA) reporter gene that is activated by rising intracellular iron levels. Treatment of these cells with doxycycline and VIT-2763 induced BLA reporter gene activity with an average $\mathrm{EC}_{50}$ of $140 \pm 50 \mathrm{nM}$, mean $\pm \mathrm{SD}$, as a consequence of increasing intracellular iron concentrations caused by blocked iron export (Figure 2E). However, the potency of hepcidin in this iron retention assay was higher with an $\mathrm{EC}_{50}$ of $39 \pm 20 \mathrm{nM}$, mean \pm SD, (Figure 2F). The effects of VIT-2763 and hepcidin were dependent of ferroportin, as shown by lack of activity when ferroportin was not induced by doxycycline addition.

In conclusion, the small molecule VIT-2763 inhibited binding of hepcidin to ferroportin, thereby preventing the internalization of fluorescently labeled hepcidin. Importantly, VIT-2763 inhibited the cellular iron efflux in a ferroportin-dependent manner and with potency comparable to hepcidin, the physiological peptide ligand of ferroportin.

VIT-2763 triggers ubiquitination and subsequent internalization and degradation of ferroportin. The generally accepted mechanism by which hepcidin regulates cellular iron export is that hepcidin binds to ferroportin, triggering its ubiquitination, internalization, and degradation, thereby blocking the iron export activity of ferroportin 
A
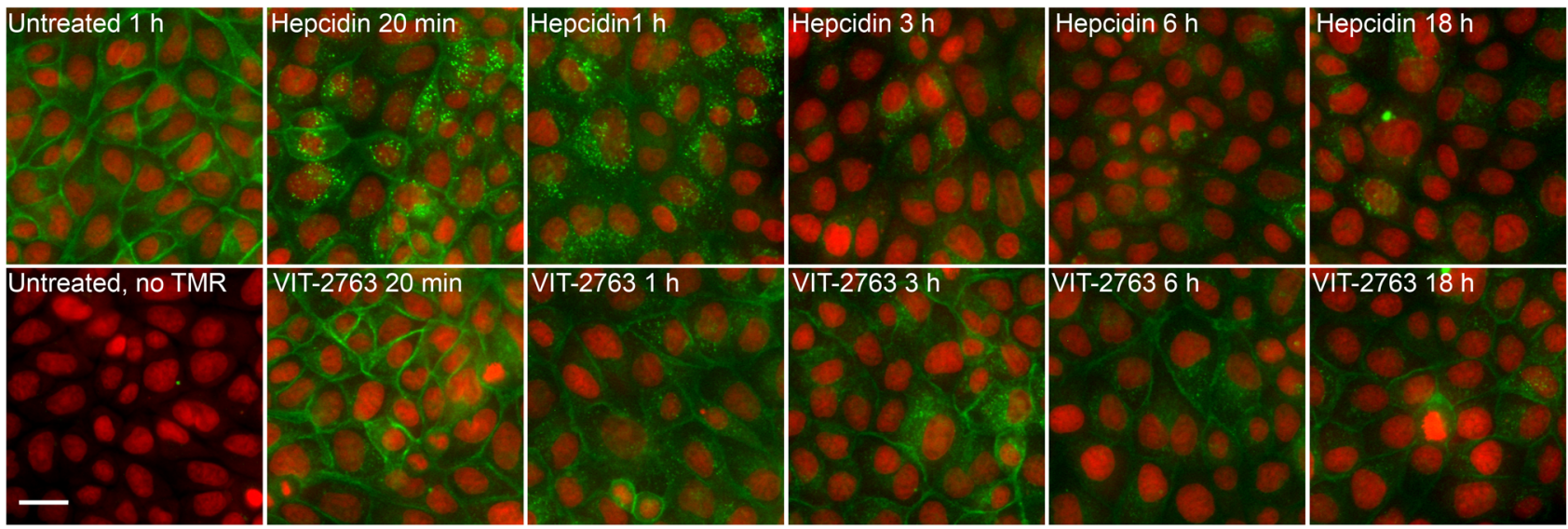

B

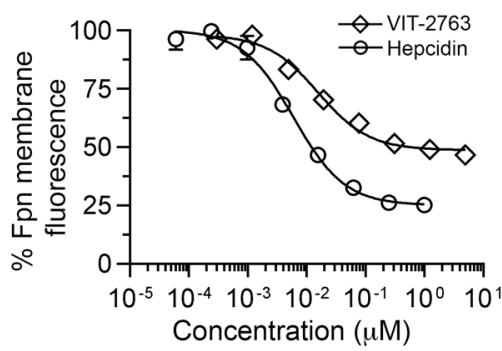

C



D$$
\text { M }
$$

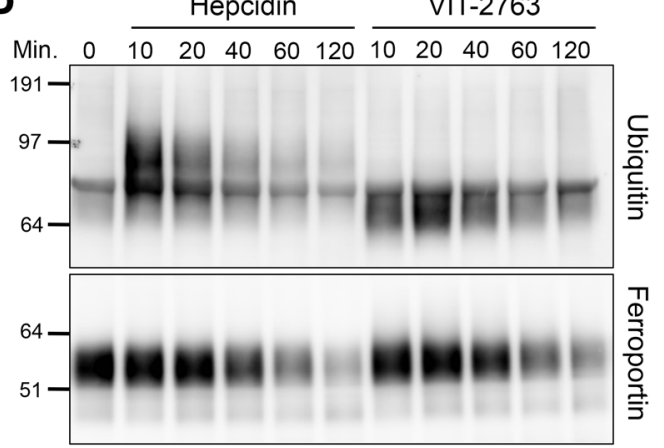

Figure 3. VIT-2763 and hepcidin induce ferroportin internalization and ubiquitination. (A) Representative images from fluorescence microscopy kinetic analysis of ferroportin internalization and degradation in MDCK cells constitutively expressing human ferroportin with a fluorescent HaloTag (green). Nuclei are depicted in red. Cells were incubated with either VIT-2763 $(20 \mu \mathrm{M})$ or hepcidin $(1 \mu \mathrm{M})$ for the indicated times. Scale bar: $25 \mu \mathrm{m}$. The full kinetic study shown in the figure was performed once. The experiment was repeated at individual time points: once at 6 hours, 3 times at 20 minutes and 1 hour, and more than 10 times at 3 hours and 18 hours with reproducible results. (B) Quantification of the ferroportin-associated membrane fluorescence in MDCK cells treated with serial dilutions of either hepcidin or VIT-2763 for 18 hours. $n=3$ per concentration. Data are shown as mean \pm SD for each concentration. (C) Kinetics of internalization of ferroportin, as depicted by decrease of membrane-associated ferroportin fluorescence in MDCK cells treated with either hepcidin $(1 \mu \mathrm{M})$ or VIT-2763 (20 $\mu \mathrm{M}) . n=2$ (1-6 hours); $n=3$ (18 hours). Mean for each time point is shown. Data in $\mathbf{B}$ and $\mathbf{C}$ are presented as mean of the percentage of ferroportin membrane fluorescence relative to untreated cells. (D) Immunoprecipitation of J774 lysates for ubiquitination and degradation studies of ferroportin. J774 cells were treated with hepcidin (150 nM) or VIT-2763 (100 nM) for 10, 20, 40, 60, or 120 minutes before harvesting and IP with MTP1 anti-ferroportin antibody. Immunoprecipitates were blotted and stained with either ubiquitin-specific (upper blot) or ferroportin-specific (lower blot) antibody (F308). The full kinetic study shown in the figure was performed once. The experiment at time points 10 and 120 minutes was performed 5 times with reproducible results.

indirectly by targeting ferroportin for degradation $(6,24,25)$. More recently, structural and mutational analyses of a bacterial ferroportin homologue (26) and mammalian ferroportin (13) suggested that hepcidin may inhibit ferroportin directly by occlusion in the absence of ferroportin endocytosis and degradation. We hypothesized that VIT-2763, despite being structurally unrelated, mimics the function of the hepcidin peptide. The TMR-hepcidin assay in J774 cells described above does not distinguish binding to ferroportin from internalization of ferroportin. To study the mechanism of action of VIT-2763 we employed 2 approaches:(a) ferroportin internalization assay with MDCK cells expressing human ferroportin with a fluorescent HaloTag; (b) immunoprecipitation studies to assess ferroportin ubiquitination in $\mathrm{J774}$ cells expressing endogenous ferroportin.

MDCK cells constitutively expressing human ferroportin fused to a HaloTag that allows labeling with the fluorescent TMR-HaloTag ligand were treated with either VIT-2763 or hepcidin for 20 minutes, 1 hour, 3 hours, 6 hours, and 18 hours (Figure
3A). Hepcidin treatment for 18 hours induced dose-dependent internalization of ferroportin in MDCK cells, as detected by disappearance of surface TMR signal by fluorescence microscopy (Figure 3B). The potency of hepcidin in the MDCK assay was higher than the potency of VIT-2763 (long-term average: hepcidin $\mathrm{EC}_{50}$ $=1.5 \pm 0.9 \mathrm{nM}$; VIT-2763 $\mathrm{EC}_{50}=14.4 \pm 8.1 \mathrm{nM}$, mean $\pm \mathrm{SD}$ ). Interestingly, the maximal response of VIT-2763 (curve span) was in average $21 \%$ lower than the response of hepcidin, suggesting that the ferroportin internalization triggered by VIT-2763 was not complete (Figure 3, A and B).

The kinetic effects of hepcidin and VIT-2763 on ferroportin expression were compared using concentrations resulting in a maximal effect for each molecule. Hepcidin $(1 \mu \mathrm{M})$ triggered ferroportin internalization and formation of intracellular vesicles starting at 20 minutes, increasing at 1 hour, and resulting in a complete ferroportin internalization from the plasma membrane at 3 hours and degradation by 3 to 6 hours (Figure 3, A-C). Remarkably, the small molecule 

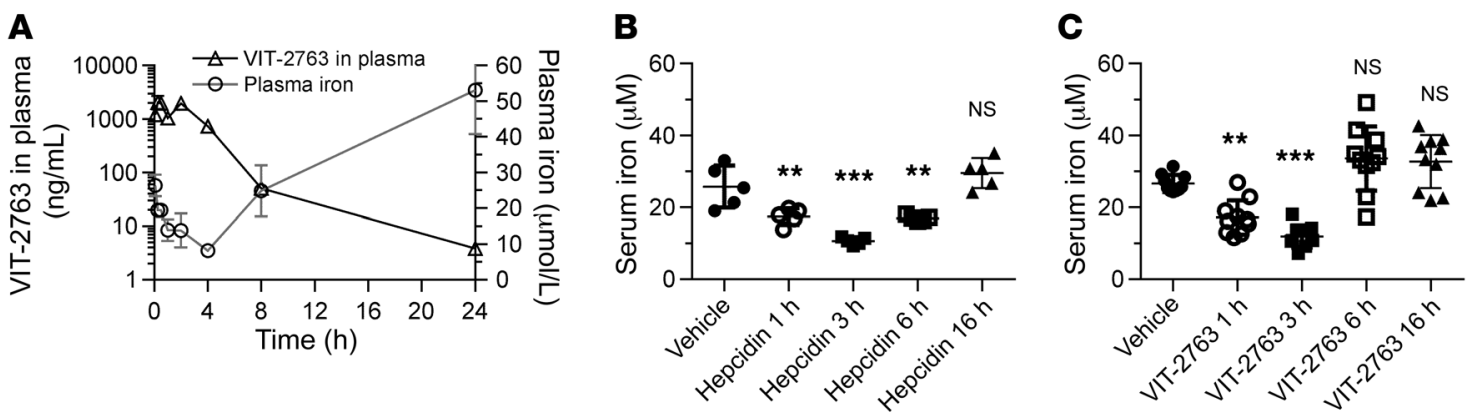

Figure 4. Rapid absorption of orally dosed VIT-2763 and decrease in serum iron induced by hepcidin and VIT-2763 in rodents. (A) Oral PK/PD of VIT-2763 at a single dose of $30 \mathrm{mg} / \mathrm{kg}$ in rats. $n=3$. Data are shown as mean \pm SD. (B and C) Serum iron levels in C57BL/ 6 mice treated with either hepcidin (5 mg/ $\mathrm{kg})(n=5)(\mathbf{B})$ or VIT-2763 (60 mg/kg) $(n=10)(\mathbf{C})$ for 1 hour, 3 hours, 6 hours, and 16 hours. Data are shown as individual values and mean \pm SD. In $\mathbf{B}$ and C, statistical analysis was performed by comparing all treatment groups to the $\mathrm{Hbb}^{\text {th } 3 /+}$ vehicle group using 1-way ANOVA with Dunnett's multiple comparison test. ${ }^{* *} P<0.01 ;{ }^{* *} P<0.001$

VIT-2763 $(20 \mu \mathrm{M})$ also induced ferroportin internalization, although with slower kinetics and incomplete disappearance of ferroportin from the plasma membrane, even after compound exposure for 18 hours (Figure 3, A-C). Interestingly, VIT-2763 caused a less prominent formation of intracellular ferroportin-containing vesicles, as compared with hepcidin, which might indicate distinct mechanisms of ferroportin trafficking and degradation (Figure 3A). Competition experiments using VIT-2763 at a constant concentration $(1 \mu \mathrm{M})$ and increasing concentrations of unlabeled hepcidin ( $4 \mathrm{nM}$ to $4 \mu \mathrm{M})$ in the MDCK cells expressing fluorescent ferroportin revealed a shift of the hepcidin dose-response curve to higher $\mathrm{EC}_{50}$ values as compared with hepcidin dose-response curve in the absence of the ferroportin inhibitor. This behavior strongly indicated that VIT-2763 competed with hepcidin for ferroportin internalization (Supplemental Figure $3, \mathrm{EC}_{50}$ [hepcidin alone] $=1.6 \mathrm{nM} ; \mathrm{EC}_{50}$ [VIT-2763 + hepcidin $]=356$ $\mathrm{nM})$. These data together with the fluorescence polarization results shown in Figure 2C suggested that VIT-2763 competes with hepcidin for ferroportin binding and internalization.

Immunoprecipitation studies performed using J774 cells expressing endogenous ferroportin showed that both VIT-2763 and hepcidin triggered ubiquitination and degradation of ferroportin. Treatment of J774 cells with hepcidin or VIT-2763 led to a rapid ubiquitination of ferroportin within 10 to 20 minutes and degradation of ferroportin starting at 40 to 120 minutes (Figure 3D). However, hepcidin induced almost full disappearance of the ferroportin signal, whereas VIT-2763 treatment for 120 minutes resulted in incomplete ferroportin degradation. In addition, hepcidin induced ferroportin ubiquitination products with higher molecular weight $(\sim 100 \mathrm{kDa})$ compared with VIT-2763 $(\sim 70 \mathrm{kDa})$, suggesting a different degree of ubiquitination (4-5 vs. 1-2 ubiquitin molecules per ferroportin, respectively).

The immunoprecipitation experiments in $\mathrm{J} 774$ cells suggested that both VIT-2763 and hepcidin utilize similar pathways of ferroportin internalization and degradation. Nevertheless, slight differences in efficacy and kinetics were demonstrated, which is in agreement with the slower kinetics and less complete degradation of ferroportin in MDCK cells expressing fluorescently labeled human ferroportin.

VIT-2763 reduced serum iron levels in rodents. To investigate the in vivo bioavailability and biological activity of VIT-2763, we determined its PK and pharmacodynamic (PD) properties after single i.v. (1 mg/kg, not shown) or oral $(30 \mathrm{mg} / \mathrm{kg}$ ) administration in male Sprague Dawley rats (Figure 4A). Orally dosed VIT-2763 showed a moderate half-life of $2.0 \pm 0.8$ hours (mean \pm SD) and good bioavailability of $48.3 \% \pm 9.9 \%$ (mean \pm SD) in rats. Injection of synthetic hepcidin in mice has been shown to decrease plasma iron levels, consistent with a blockade of iron absorption and iron export from tissue stores and from macrophages involved in iron recycling (12). Plasma iron was measured as a marker for PD activity of VIT-2763 in vivo. Plasma iron in rats dosed orally with VIT-2763 decreased over time, reaching minimal levels 4 hours after oral dosing and recovering within 8 to 24 hours. Interestingly, plasma iron levels at 24 hours were higher than at baseline ( 5 minutes after dose), most likely due to a regulatory feedback response to hypoferremia (Figure 4A).

To compare the efficacy of hepcidin and VIT-2763 in vivo, an acute model of hypoferremia in C57BL/6 mice was used. Injection of synthetic human hepcidin i.p. (5 mg/kg) into mice resulted in a significant reduction of serum iron levels at 1 hour, 3 hours, and 6 hours $(32 \%, 59 \%$, and $34 \%$ lower than the vehicle, respectively, Figure 4B). VIT-2763 dosed orally at $60 \mathrm{mg} / \mathrm{kg}$ rapidly decreased serum iron at 1 hour and 3 hours to levels comparable to those seen with hepcidin ( $40 \%$ and $58 \%$ lower than the vehicle), demonstrating similar effects of VIT-2763 and hepcidin in C57BL/6 mice from 1 hour to 3 hours after dosing (Figure 4, B and C). Serum iron levels in mice treated with VIT-2763 for 6 hours did not change significantly compared with those in the vehicle group. Both, hepcidin and VIT-2763 had no effect on serum iron levels at 16 hours after dose was given.

VIT-2763 decreased iron overload in the Hbb ${ }^{\text {th } 3 /+}$ mouse model of $\beta$-thalassemia intermedia. Parenteral administration of agents that induce the synthesis of endogenous hepcidin or modified hepcidin peptides has been shown to correct ineffective erythropoiesis and to ameliorate iron overload in the $\mathrm{Hbb}^{\mathrm{th} 3 /+}$ model of $\beta$-thalassemia intermedia (18-20). Based on the similar mode of action of VIT-2763 and hepcidin in cell-based and biophysical assays, as well as acute efficacy in WT rodents, we hypothesized that VIT-2763 might improve the pathophysiological parameters in the $\mathrm{Hbb}^{\mathrm{th} 3 /+}$ model of $\beta$-thalassemia intermedia and provide a preclinical proof of concept for development of oral ferroportin inhibitor for therapy of $\beta$-thalassemia. 
A

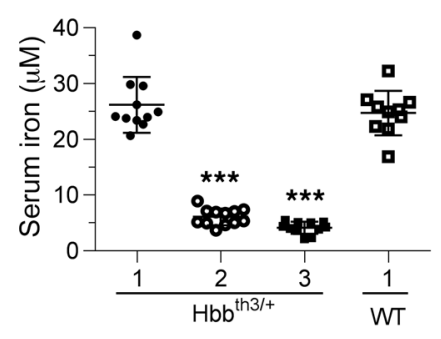

D

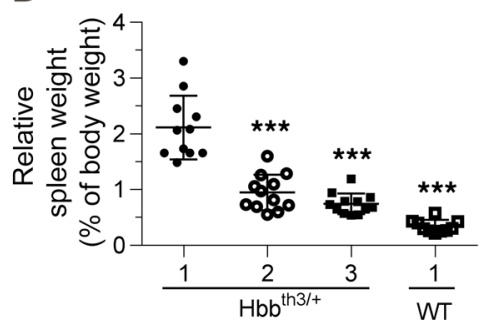

B

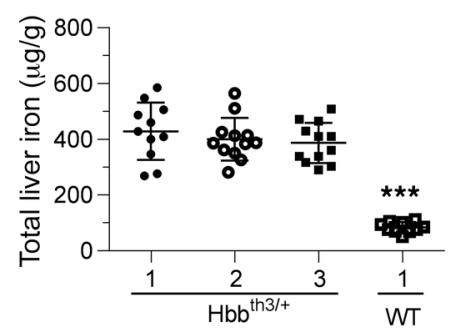

E

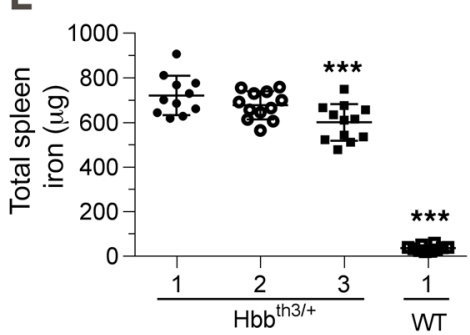

C

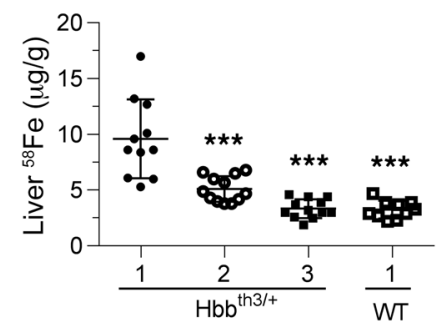

F

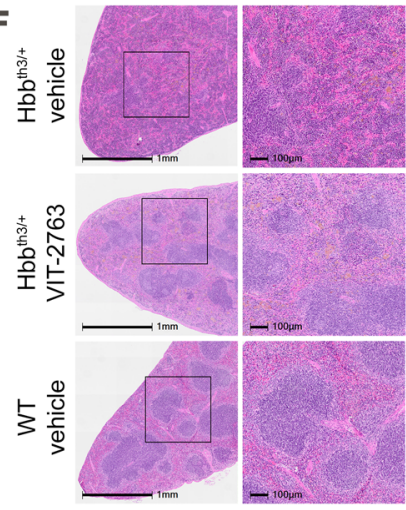



Figure 5. VIT-2763 decreased serum iron and prevented liver iron loading in Hbb $\mathbf{b}^{\text {th } 3 /+}$ mice. (A) VIT-2763 significantly decreased serum iron levels in Hbb th3/+ mice 3 hours after oral dosing at study day 36. (B) Total liver iron concentration remained unchanged following 36 days of treatment with VIT-2763. (C) VIT2763 prevented liver ${ }^{58} \mathrm{Fe}$ loading in $\mathrm{Hbb}^{\text {th } 3 /+}$ mice. (D) VIT-2763 reduced the relative spleen weight of $\mathrm{Hbb}^{\text {th } 3 /+}$ mice. (E) Effect of VIT-2763 on total spleen iron content. (A-E) x axis labels: 1, vehicle; 2, VIT-2763 (30 mg/kg); 3, VIT-2763 (100 mg/kg). Individual values and mean \pm SD are shown. Statistical analysis was performed by comparing all treatment groups to the $\mathrm{Hbb}^{\text {th } 3 /+}$ vehicle group using 1-way ANOVA with Dunnett's multiple comparison test. $n=10-12$. (F) Representative photographs from H\&E (left) and DAB-enhanced Perls staining (right) in spleen sections from vehicle-treated (top) or VIT-2763-treated (100 mg/kg, middle) $\mathrm{Hbb}^{\text {th } 3 /+}$ mice and vehicle-treated WT mice (bottom). Shown are representative photographs from 10 to 12 mice per group and 3 sections from each spleen. Scale bars: $1 \mathrm{~mm}$ in originals, $100 \mu \mathrm{m}$ in enlargements. ${ }^{* *} P<0.001$.

$\mathrm{Hbb}^{\text {th } 3 /+}$ mice absorb excessive amounts of iron as a consequence of inadequately low hepcidin levels relative to the high iron content in liver, spleen, and kidney and increased ferroportin expression in the duodenum (27). To investigate the efficacy of VIT-2763 in the $\mathrm{Hbb}^{\text {th } 3 /+}$ model, mice were fed a diet with a low amount of iron $(<10 \mathrm{mg} / \mathrm{kg})$ and dosed orally with either 30 or $100 \mathrm{mg} / \mathrm{kg}$ VIT-2763 or vehicle twice daily (bid) for 36 days. In between compound doses, mice had access to drinking water containing the stable iron isotope ${ }^{58} \mathrm{Fe}$, which allowed differentiating iron absorbed during and before the study. Treatment of $\mathrm{Hbb}^{\mathrm{th} 3 /+}$ mice with 30 or $100 \mathrm{mg} / \mathrm{kg}$ VIT-2763 significantly decreased serum iron levels, by $77 \%$ and $84 \%$, relative to the vehicle $\mathrm{Hbb}^{\mathrm{th} 3 /+}$ group, respectively (Figure $5 \mathrm{~A}$ ), demonstrating the acute effect of the compound on systemic iron. As expected, by blocking ferroportin on liver macrophages and hepatocytes, VIT-2763 did not change the total liver iron. However, VIT-2763 very efficiently prevented additional liver iron loading during the study, as shown by a dose-dependent reduction of ${ }^{58} \mathrm{Fe}$ concentration in liver (Figure 5, B and C).

The ineffective erythropoiesis in $\mathrm{Hbb}^{\mathrm{th} /+}$ mice causes excessive proliferation of erythroid precursors and expansion of the red pulp in the spleen, leading to splenomegaly. Treatment of $\mathrm{Hbb}^{\text {th } 3 /+}$ mice with VIT-2763 resulted in a significant reduction of the relative spleen weight, by $52 \%$ and $65 \%$ of the $\mathrm{Hbb}^{\text {th } 3 /+}$ vehicle control for 30 and $100 \mathrm{mg} / \mathrm{kg}$ VIT-2763, respectively (Figure 5D). Furthermore, $\mathrm{Hbb}^{\text {th } 3 /+}$ mice receiving VIT-2763 showed improved distribution of spleen red and white pulp compartments and had a less diffuse iron-staining pattern compared with $\mathrm{Hbb}^{\mathrm{th} 3 /+}$ mice treated with vehicle (Figure $5 \mathrm{~F}$ ). These results highlight the potential of the oral ferroportin inhibitor to attenuate excessive extramedullar erythropoiesis in spleen. The concentration of spleen iron increased dose dependently (not shown), consistent with the decrease in the spleen weight and retention of iron in spleen macrophages; however, the total spleen iron content remained unchanged in the group treated with $30 \mathrm{mg} / \mathrm{kg}$ and decreased significantly in mice treated with the $100 \mathrm{mg} / \mathrm{kg}$ compound (Figure 5E).

VIT-2763 ameliorated anemia and improved ineffective erythropoiesis in $\mathrm{Hbb}^{t h 3 /+}$ mice. Notably, ferroportin inhibition with the oral drug VIT-2763 not only decreased plasma and organ iron levels, but also improved the hematological parameters in $\mathrm{Hbb}^{\mathrm{th} 3 /+}$ mice. VIT-2763 significantly increased Hb levels (as of day 8 of treatment; Figure 6A), RBC counts (Figure 6B), mean corpuscular $\mathrm{Hb}$ concentration (MCHC) (Figure 6C), and significantly lowered reticulocyte counts (Figure 6D), mean corpuscular $\mathrm{Hb}(\mathrm{MCH})$ (Figure 6E), mean corpuscular volume (MCV) (Figure 6F), and RBC distribution width (RDW) (Figure 6G) in $\mathrm{Hbb}^{\mathrm{th} 3 /+}$ mice, as compared with the $\mathrm{Hbb}^{\mathrm{th} 3 /+}$ vehicle group. Therefore, ferroportin inhibition by VIT-2763 significantly (Figure 6) ameliorated anemia and corrected pathologically altered RBCs and reticulocyte counts, indicating improved erythropoiesis in $\mathrm{Hbb}^{\mathrm{th} /+}$ mice. Remarkably, dietary iron restriction of $\mathrm{Hbb}^{\mathrm{th} 3 /+}$ mice for the dura- 
A

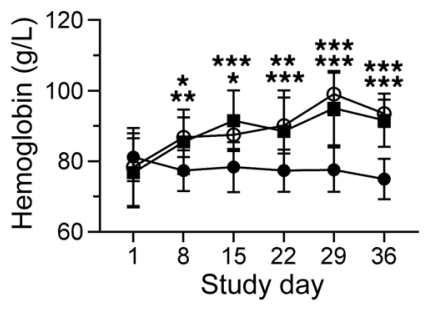

B

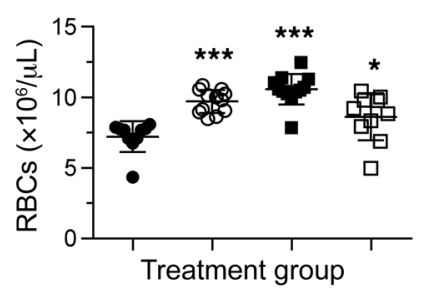

D

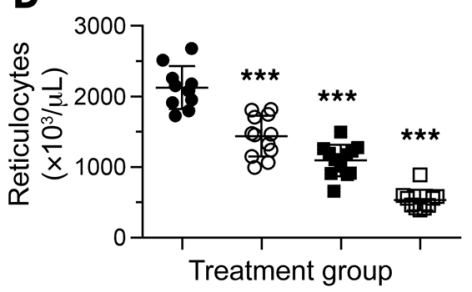

$\mathbf{F}$

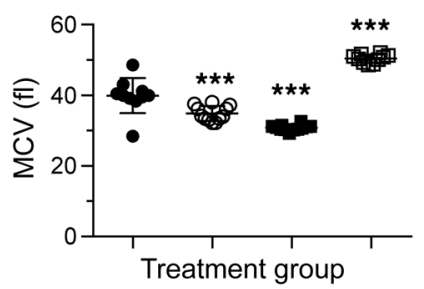

- $\mathrm{Hbb}^{\text {th } 3 /+}$ vehicle

O Hbb ${ }^{\text {th } 3 /+}$ VIT-2763 $(30 \mathrm{mg} / \mathrm{kg})$

- Hbb $^{\text {th } 3 /+}$ VIT-2763 (100 mg/kg)

$\square$ WT vehicle



E

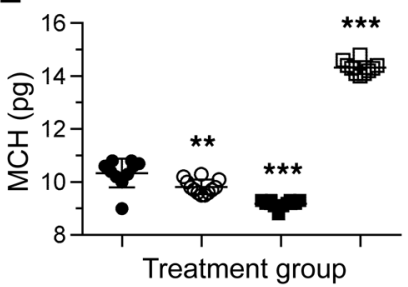

G

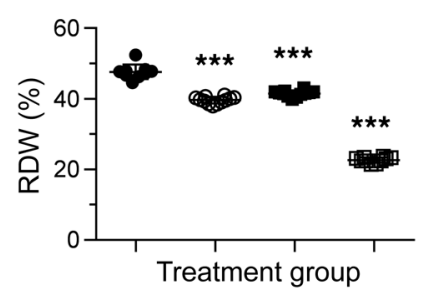

Figure 6. VIT-2763 significantly corrected anemia and improved RBC parameters in $\mathbf{H b b}^{\text {th } 3 /+}$ mice. (A) VIT-2763 significantly increased $\mathrm{Hb}$ concentration starting at day 8 of dosing in $\mathrm{Hbb}^{\text {th } 3 /+}$ mice. Mean $\pm \mathrm{SD}$ values of $\mathrm{Hb}$ concentrations are shown. Statistical analysis was performed using repeated measures 2-way ANOVA with Dunnett's multiple comparison test to compare all treatment groups to the $\mathrm{Hbb}^{\mathrm{th} 3 /+}$ vehicle group over time. $n=10-12$ mice. At the study end, VIT-2763 increased RBC counts (B) and MCHC (C) and decreased reticulocyte counts (D), MCH (E), MCV (F), and RDW (G) in $\mathrm{Hbb}^{\text {th } 3 /+}$ mice. For B-F, individual values and mean $\pm S D$ are shown. Statistical analysis was performed by comparing all treatment groups to the $\mathrm{Hbb}^{\text {th } 3 /+}$ vehicle group using 1-way ANOVA with Dunnett's multiple comparison test. $n=10-12$ mice. ${ }^{*} P<0.05 ;{ }^{* *} P<0.01 ;{ }^{* *} P<0.001$.

$\mathrm{C}$ and $\mathrm{F}$ ), indicating diminished oxidative damage of erythroid cells. As a result, mature RBC and their precursors in blood of $\mathrm{Hbb}^{\text {th } 3 /+}$ mice receiving VIT-2763 expressed less phosphatidylserine (PS), indicating reduced apoptosis (Supplemental Figure 4). The increased proportion of mature erythroid cells and decreased oxidative stress in BM and spleens of $\mathrm{Hbb}^{\mathrm{th} 3 /+}$ mice together with the reduced apoptosis of mature RBCs and $\mathrm{RBC}$ precursors in blood suggest that VIT-2763 ameliorated the ineffective erythropoiesis in $\mathrm{Hbb}^{\mathrm{th} /+}$ mice.

VIT-2763 reduced the formation of $\alpha$-globin aggregates and extended the life span and improved the functional parameters of circulating RBCs in $\mathrm{Hbb}^{\text {th } 3 /+}$ mice. To investigate how VIT-2763 improves anemia and iron overload, $\mathrm{Hbb}^{\mathrm{th} /+}$ mice were treated with the compound for 4 to 7 weeks and a broader range of biomarkers was investigated in several independent studies.

In $\beta$-thalassemia intermedia, the imbalanced synthesis of $\alpha$ - and $\beta$-globin chains of $\mathrm{Hb}$ leads to formation of insoluble $\alpha$-globin aggregates containing free heme and iron, causing ROS formation and apoptosis of the late-stage erythroid progenitors. Analysis of the membrane-bound globins by triton acetic acid urea (TAU) gel electrophoresis showed a dosedependent decrease in the levels of toxic $\alpha$-globin aggregates in $\mathrm{RBC}$ membrane preparations from $\mathrm{Hbb}^{\mathrm{th} / /+}$ mice treated with VIT2763 for 28 days compared with the $\mathrm{Hbb}^{\text {th } 3 /+}$ vehicle group (Figure $8 \mathrm{~A})$. The reduction of the membrane $\alpha$-globin was present as early as day 8 of compound treatment (data not shown) and further decreased in the following 2 weeks, suggesting a rapid improvement of erythropoiesis. The insoluble globin aggregates on RBC membranes contain products of $\mathrm{Hb}$ oxidative denaturation, such as metHb, free heme, and iron, which all contribute to ROS generation in RBCs (28). In agreement with the reduced $\alpha$-globin aggregates in RBCs, VIT-2763 decreased the percentage of ROSpositive RBCs in $\mathrm{Hbb}^{\mathrm{th} 3 /+}$ mice from $67 \%$ to $30 \%$ (Figure $8 \mathrm{~B}$ ).

Reticulocytes of healthy individuals clear mitochondria by mitophagy during maturation, and terminally differentiated RBCs produce energy by glycolysis (29). However, it has been shown that mitophagy in $\beta$-thalassemia is impaired, resulting in retention of mitochondria in mature RBCs $(30,31)$. Flow cytometry analysis of MitoTracker-labeled blood cells showed that essentially all reticulocytes from both WT and $\mathrm{Hbb}^{\mathrm{th} /+}$ mice contained mitochondria (MitoTracker ${ }^{+}$cells, Figure 9A). Upon maturation, RBCs from WT mice progressively eliminated their mitochondria, with only $1.5 \%$ of terminally differentiated RBCs retaining of mature BM and spleen erythrocytes stained positive for ROS in VIT-2763- compared with vehicle-treated $\mathrm{Hbb}^{\text {th } 3 /+}$ mice (Figure 7, 
A
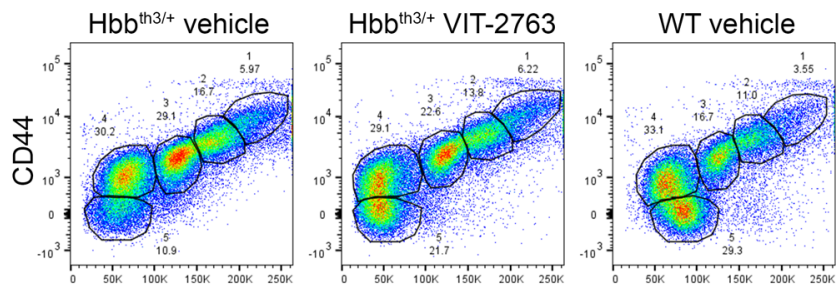

FCS-A

B
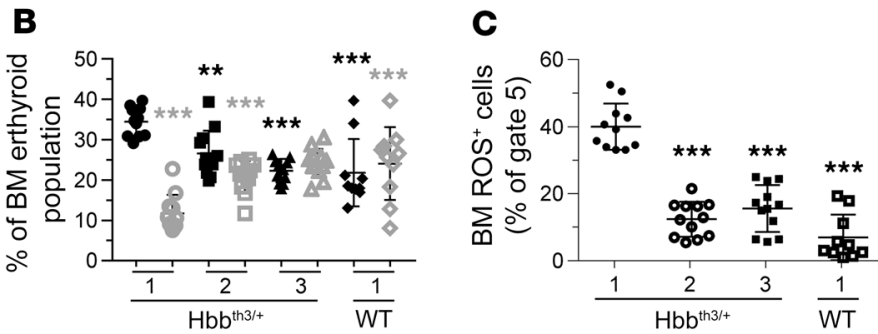

D
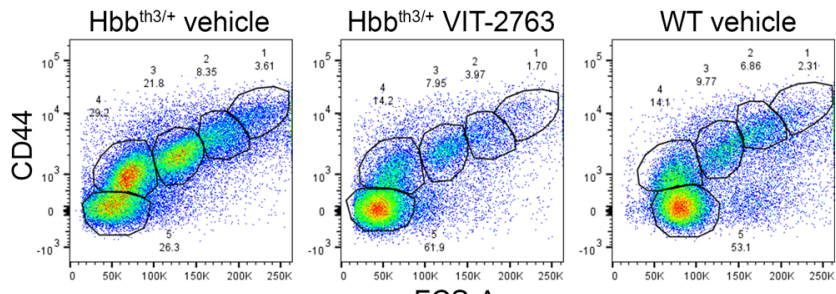

FCS-A
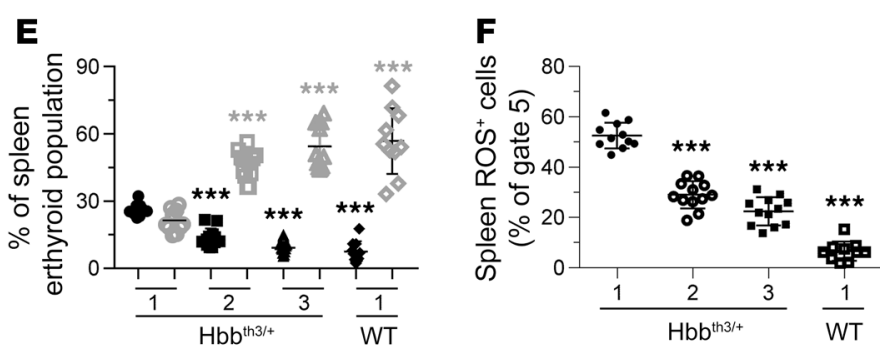

mitochondria (Figure 9, A and B). Strikingly, 10.3\% (group average value) of mature RBCs from the $\mathrm{Hbb}^{\mathrm{th} 3 /+}$ vehicle group preserved mitochondria and produced higher levels of ROS compared with RBCs without mitochondria (ratio of MFI of ROSpos MitoTracker ${ }^{\text {pos }}$ to ROS $^{\text {pos }}$ MitoTracker ${ }^{\text {neg }} \mathrm{RBCs}=2.5$, group average value, representative dot plot shown in Figure 9A). In $\mathrm{Hbb}^{\text {th } 3 /+}$ mice, VIT-2763 significantly reduced the proportion of mature RBCs containing mitochondria to an average of $4.9 \%$ (Figure 9B). Importantly, RBCs devoid of mitochondria produced lower levels of ROS (ROS ${ }^{\text {pos }}$ MitoTracker ${ }^{\text {neg }}$ populations in Figure 9A). These data suggested that at least part of the oxidative stress in RBCs from $\mathrm{Hbb}^{\mathrm{th} 3 /+}$ mice is mediated by erythrocytes that failed to eliminate their mitochondria. By improving erythropoiesis, VIT-2763 indirectly ameliorated mitophagy and decreased the oxidative stress in $\mathrm{RBCs}$ of $\mathrm{Hbb}^{\mathrm{th} 3 /+}$ mice.

Increased ROS levels in RBCs of $\mathrm{Hbb}^{\mathrm{th} 3 /+}$ mice cause membrane damage and lead to exposure of PS to the extracellular space. PS exposure to the outer cell membrane is an apoptotic signal and targets RBCs for phagocytosis (32). VIT-2763 treatment of $\mathrm{Hbb}^{\mathrm{th} /+}$ mice significantly decreased PS exposure of RBCs and precursors of mature erythrocytes, as shown by annexin $\mathrm{V}$ staining (Figure 10A and Supplemental Figure 4). PS exposure marks
Figure 7. VIT-2763 treatment improved the ineffective erythropoiesis in $\mathrm{BM}$ and spleen of $\mathbf{H b}^{\mathrm{th} 3 /+}$ mice. Gating strategy used to identify erythroid progenitors in BM (A) or spleen (D) by flow cytometry. Representative dot plots from 1 out of 4 independent experiments showing vehicle- or VIT-2763-treated Hbb ${ }^{\text {th } 3 /+}$ and WT mice. VIT-2763 decreased the frequency of polychromatic erythroblasts (population in gate 3 ) in BM (B) and spleen (E). VIT-2763 treatment reduced the percentages of BM (C) and spleen (F) ROS-positive mature erythrocytes. (B and E) Black symbols show polychromatic erythroblasts, and gray symbols show mature erythrocytes. (B, C, E and F) $x$ axis labels: 1, vehicle; 2, VIT-2763 (30 mg/kg); 3, VIT-2763 (100 mg/kg). Individual values and mean \pm SD are shown. Statistical analysis was performed by comparing all treatment groups to the $\mathrm{Hbb}^{\text {th } 3 /+}$ vehicle group using 1-way ANOVA with Dunnett's multiple comparison test. $n=10-12$ mice. ${ }^{* *} P<0.01$; ${ }^{* *} P<0.001$.

RBCs for phagocytosis by scavenging macrophages that deliver the damaged RBCs to spleen and liver for degradation (33). The released heme is catabolized by heme oxygenase 1 (HMOX1), which is upregulated in $\beta$-thalassemia due to excessive RBC apoptosis (34). VIT-2763 treatment significantly reduced the expression of $\mathrm{Hmox} 1$ in livers of $\mathrm{Hbb}^{\text {th } 3 /+}$ mice, most likely as a result of the decreased RBC turnover (Figure 10B). Indeed, the half-life of RBCs in $\mathrm{Hbb}^{\text {th3/+ }}$ mice treated with vehicle was 8 days and administration of VIT-2763 extended it to 22 days, which was similar to the half-life of RBCs in WT mice (Figure 10C).

VIT-2763 ameliorated hypoxia in $\mathrm{Hbb}^{\text {th } 3 /+}$ mice. $\mathrm{Hbb}^{\mathrm{th} 3 /+}$ mice are systemically hypoxic as a result of inappropriate tissue oxygenation by the defective RBCs $(14,18)$. Indeed, flow cytometry analysis of RBCs stained with a fluorescent surrogate marker for hypoxia (Enzo Life Sciences) confirmed a higher MFI signal in $\mathrm{Hbb}^{\mathrm{th} 3 /+}$ mice treated with vehicle compared with WT mice (Figure 11A). RBCs from $\mathrm{Hbb}^{\text {th } 3 /+}$ mice that received VIT-2763 for 4 weeks showed reduced MFI levels of the fluorogenic hypoxia marker, suggesting improved oxygenation of RBCs (Figure 11A). Hypoxia is in the center of the vicious circle driving iron overabsorption and ineffective erythropoiesis in $\beta$-thalassemia by upregulating genes involved in iron uptake and erythropoiesis, such as DMT1, DcytB, ferroportin, and EPO. The effect of VIT-2763 on the hypoxia-sensitive marker in RBCs is most likely indirect and mediated by the improved erythropoiesis and increased $\mathrm{Hb}$ resulting in a better tissue oxygenation. Consistent with the improvement of tissue oxygenation, $\mathrm{Hbb}^{\text {th } 3 /+}$ mice treated with VIT-2763 produced significantly less serum EPO compared with the vehicle group (Figure 11B).

Eleavated EPO levels in $\mathrm{Hbb}^{\text {th } 3 /+}$ mice upregulated Erfe, an erythroid regulatory hormone known to suppress hepcidin (17). In agreement with reduced serum EPO, Erfe gene expression was significantly lower in spleens of $\mathrm{Hbb}^{\mathrm{th} 3 /+}$ mice dosed with VIT-2763 compared with those treated with vehicle alone (Figure 11C). Erfe is produced by erythrocyte precursors proliferating massively in spleens of $\mathrm{Hbb}^{\mathrm{th} 3 /+}$ mice as a consequence of extramedullar erythropoiesis. Therefore, the effect of VIT-2763 on Erfe expression in spleen is mediated by reduced numbers of $\mathrm{RBC}$ precursors in the spleen.

Liver hepcidin (Hamp) expression in $\mathrm{Hbb}^{\text {th } 3 /+}$ and WT mice was similar and did not change upon VIT-2763 treatment (Figure 11D), as similarly reported for minihepcidin in $\mathrm{Hbb}^{\mathrm{th} 3 / \mathrm{t}}$ mice (18). 


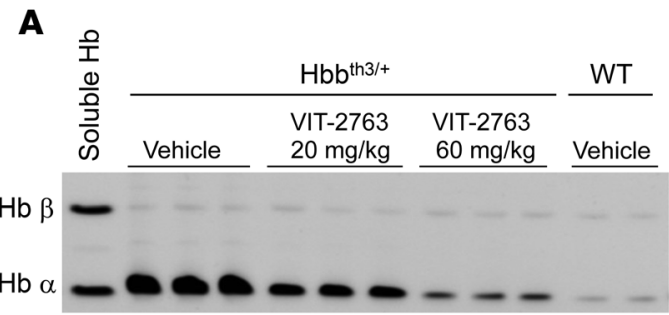

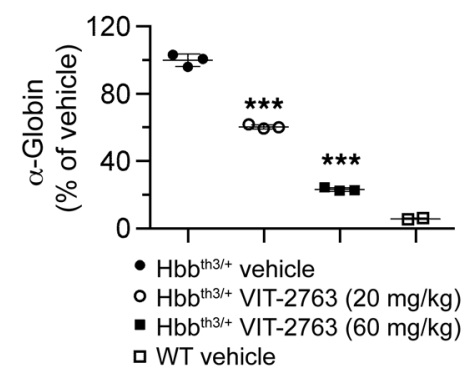

B



Figure 8. VIT-2763 reduced the formation of insoluble $\alpha$-globin aggregates in RBCs of $\mathbf{H b b}^{\text {thl/+ }}$ mice. (A) TAU gel electrophoresis of membrane-bound globins in RBCs from Hbb ${ }^{\text {th } 3 /+}$ and WT mice. $n=4-6$. Each band is a pool of samples from 2 mice. Soluble $\alpha$ and $\beta$ Hb from WT RBCs are shown as a reference. Quantification of the signal intensity of the TAU gel $\alpha$-globin bands by densitometry is shown next to the TAU gel picture. Similar effect of VIT-2763 on $\alpha$-globin was documented in 4 independent experiments. (B) VIT-2763 (60 mg/kg bid for 28 days) reduced the proportion of ROS ${ }^{+}$Ter119+ RBCs of Hbb ${ }^{\text {th } 3 /+}$ mice. Individual values and mean \pm SD are shown. Statistical analysis was performed by comparing all treatment groups to the Hbb ${ }^{\text {th } 3 /+}$ vehicle group using 1 -way ANOVA with Dunnett's multiple comparison test. $n=9-11 .{ }^{* *} P<0.001$.

Effects of VIT-2763 on myeloid precursors in spleens of $\mathrm{Hbb}^{\text {th } 3 /+}$ mice. It has been previously shown that the proportion of immature myeloid cells is highly increased in spleens of $\mathrm{Hbb}^{\mathrm{th} 3 /+}$ mice compared with WT, which suggested that the terminal neutrophil maturation in spleens of $\mathrm{Hbb}^{\mathrm{th} 3 /+}$ mice is compromised (35). Flow cytometry analysis of myelopoiesis in spleens confirmed a significant expansion of mature neutrophils (Figure 12A, population i), immature myeloid cells (Figure 12, population ii) and inflammatory monocytes (Figure 12A, population iv) in $\mathrm{Hbb}^{\text {th } 3 /+}$ mice. VIT-2763 dosed for 3 weeks did not change the percentage of resident monocytes (population iii), however VIT-2763 significantly reduced the percentage of mature neutrophils (population i), immature myeloid cells (population ii) and inflammatory monocytes (population iv, Figure 12, A and B). These data demonstrated that iron restriction by the ferroportin inhibitor VIT-2763 results not only in correction of erythropoiesis, but also leads to amelioration of myelopoiesis in spleen. It remains to be investigated whether the quantitative changes in neutrophil populations also translate to functional improvement, i.e., chemotaxis, opsonization, and ROS production in response to bacterial challenge.

\section{Discussion}

The data in this paper summarize the discovery and preclinical efficacy of the oral ferroportin inhibitor VIT-2763. This small molecule drug has a mode of action similar to that of the peptide hepcidin: in cells, VIT-2763 blocked iron efflux with a potency similar to that of hepcidin, competed with hepcidin for ferroportin binding, and triggered ferroportin internalization and ubiquitination. Importantly, VIT-2763 improved the ineffective erythropoiesis, ameliorated ane$\mathrm{mia}$, and prevented liver iron loading in the $\mathrm{Hbb}^{\mathrm{th} 3 /+}$ mouse model
A
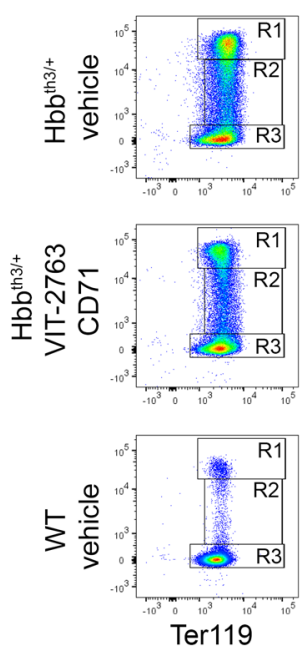

Reticulocytes (R1)
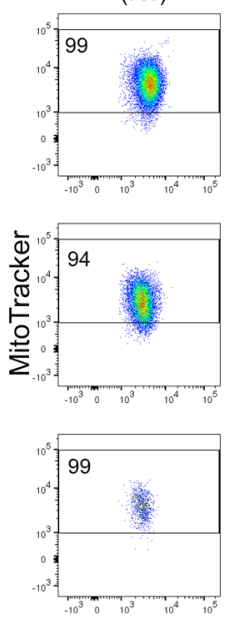

RBC precursors (R2)
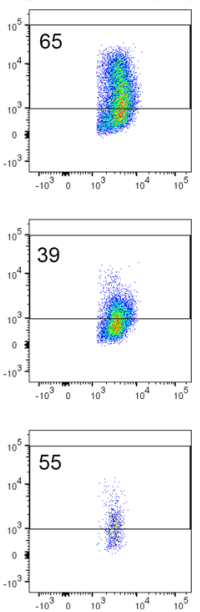

Ter119
Mature RBC (R3)
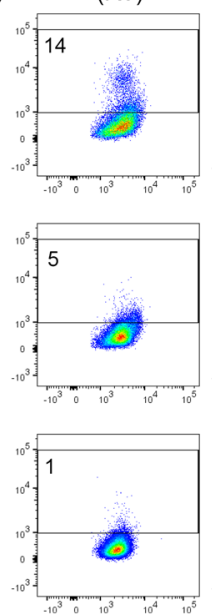

Mature RBC MitoTr. vs. ROS
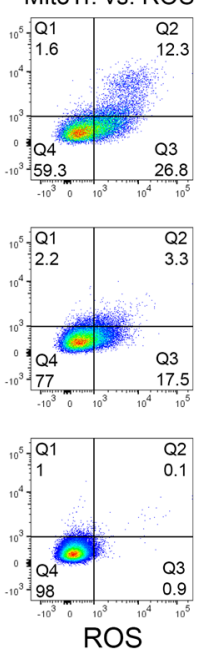

B

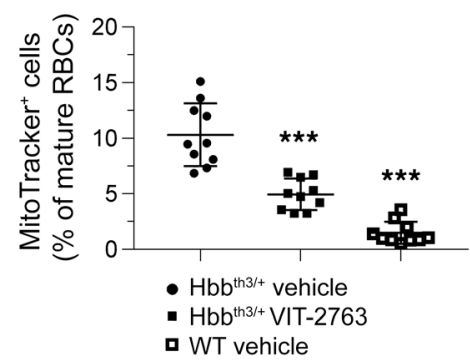

Figure 9. VIT-2763 improved the elimination of mitochondria in RBCs of Hbb $\mathbf{b}^{\text {th } 3 /+}$ mice. (A and B) Mitochondria are retained in mature RBCs of Hbb ${ }^{\text {th } 3 /+}$ mice and cleared in mature RBCs of $\mathrm{Hbb}^{\text {th } 3 /+}$ mice treated with VIT-2763. (A) Flow cytometry analysis showing representative dot plots from 1 out of 3 inde-

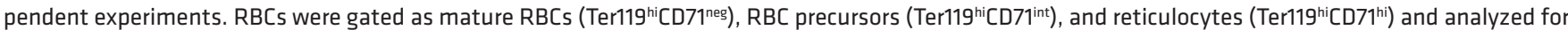
mitochondrial labeling by MitoTracker Deep Red staining and ROS by CM-H2DCFDA staining. (B) Quantification of the percentage of RBCs with mitochondria. Individual values and mean \pm SD are shown. Statistical analysis was performed by comparing all treatment groups to the $\mathrm{Hbb}^{\text {th } 3 /+}$ vehicle group using 1-way ANOVA with Dunnett's multiple comparison test. $n=10$ mice. ${ }^{* * *} P<0.001$. 
A

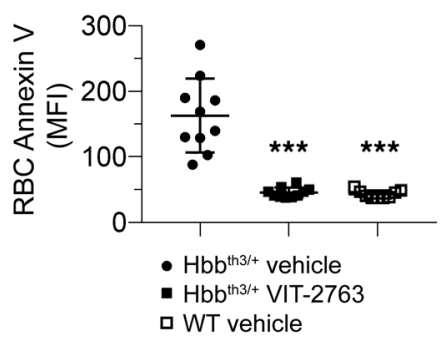

B

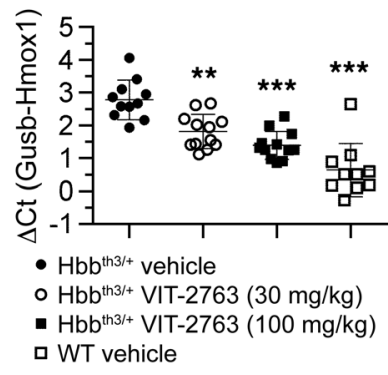

C

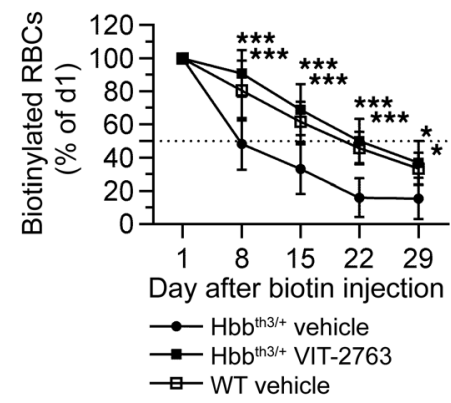

Figure 10. VIT-2763 decreased apoptosis and extended the life span of RBCs in Hbb ${ }^{\text {th } 3 /+}$ mice. (A) VIT-2763 lowered PS exposure on peripheral RBCs, as detected by decrease in intensity of annexin V staining. $n=9-10$. (B) VIT-2763 reduced the expression of liver Hmox1, as detected by qPCR. $n=10-12$. (A and B) Individual values and mean \pm SD are shown. Statistical analysis was performed by comparing all treatment groups to the Hbb ${ }^{\text {th } 3 /+}$ vehicle group using 1-way ANOVA with Dunnett's multiple comparison test. (C) VIT-2763 (60 mg/kg for 7 weeks) extended the life span of RBCs in Hbb ${ }^{\text {th } 3 /+}$ mice. Biotin labeling was performed after 21 days of dosing with VIT-2763. Shown is the percentage of biotinylated Ter119+ cells normalized to the percentage of labeled cells at day 1 after biotin injection. $n=4-10$. Mean \pm SD values are shown. Statistical analysis was performed using repeated measures 2-way ANOVA with Dunnett's multiple comparison test to compare all treatment groups to the $\mathrm{Hbb}{ }^{\text {th } 3 /+}$ vehicle group over time. ${ }^{*} P<0.05$; ${ }^{* *} P<0.01 ;{ }^{* *} P<0.001$.

of $\beta$-thalassemia. Presumably, VIT-2763 limits iron availability for formation of toxic $\alpha$-globin aggregates and ROS in erythroid precursors and thereby improves erythropoiesis. As a result, more RBCs with extended life span ameliorate anemia and improve tissue oxygenation. VIT-2763 not only improved erythropoiesis, but also corrected the proportion of spleen myeloid precursors in $\mathrm{Hbb}^{\text {th } 3 /+}$ mice. VIT-2763 showed no cytotoxicity in assay detecting cellular ATP levels $\left(\mathrm{IC}_{50}>100 \mu \mathrm{M}\right)$. In nonclinical toxicology studies in rodents, the compound was well tolerated, with no observed adverse effect level (NOAEL) above $600 \mathrm{mg} / \mathrm{kg}$ in 14-day dosing studies with healthy rodents. In longer-term studies, there was no dose-limiting toxicity. The dose-limiting effects in healthy rodents were related to the pharmacology of VIT-2763 (restricting iron uptake) and the expected iron deficiency anemia and effects were secondary to that.
Based on the mode of action described here, VIT-2763 is expected to correct ineffective erythropoiesis and iron overload in a range of diseases, such as hereditary hemochromatosis and hereditary anemias, e.g., thalassemia and sickle cell disease, or other hemoglobinopathies. In addition, VIT-2763 is expected to ameliorate myeloproliferative/myelodysplastic disorders, such as polycythemia vera and myelodysplastic syndrome (MDS). In a recently concluded phase I study in healthy individuals, VIT-2763 was well tolerated and showed a dose-linear PK profile. Importantly, serum iron was lowered and remained below baseline values up to 24 hours after the dose. Following these positive phase I results, Vifor Pharma intends to start a phase II proof-of-concept trial in $\beta$-thalassemia patients (22).

All hepcidin-mimetics in clinical development, such as synthetic hepcidin, hepcidin peptidomimetics, or agents that
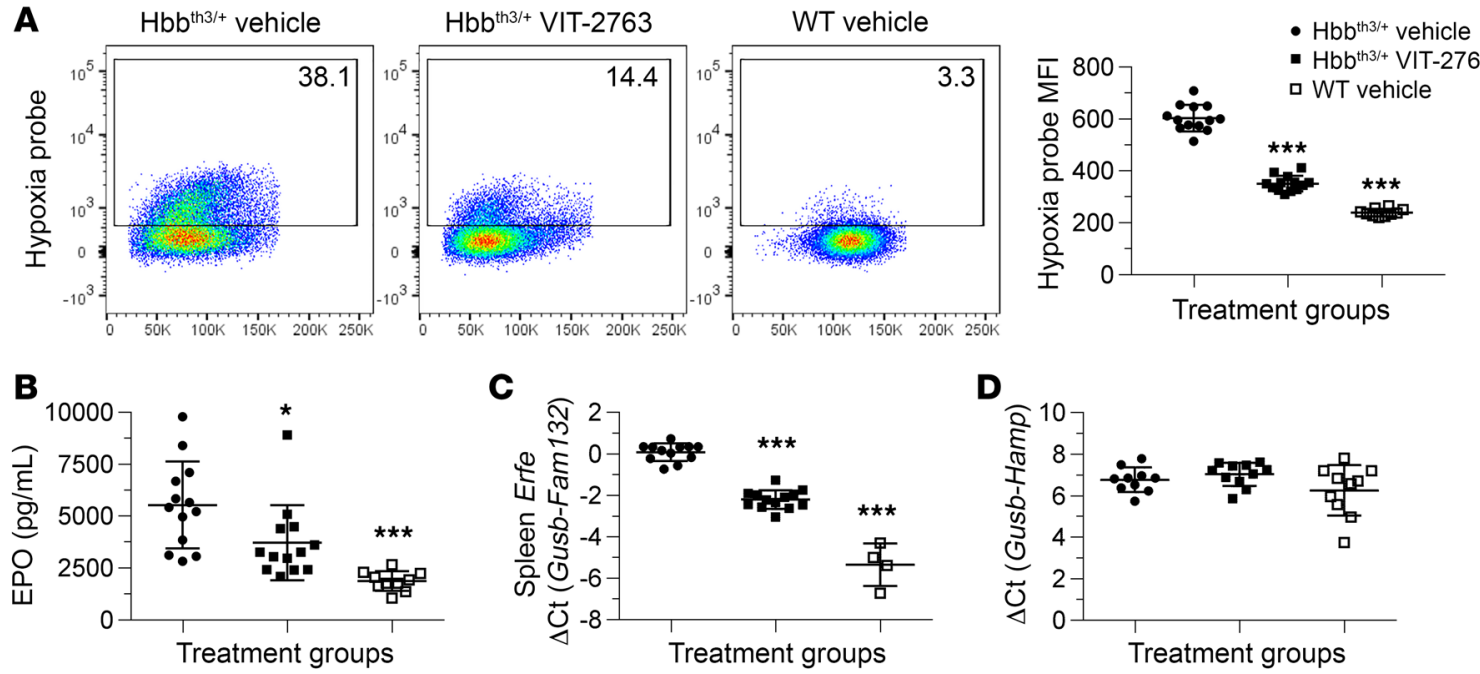

Figure 11. VIT-2763 reduced hypoxia response in RBCs, excessive serum EPO, and Erfe expression in spleens of Hbb ${ }^{\text {th } 3 /+}$ mice without effect on liver Hamp. (A) Percentage of hypoxic RBCs (left, dot plots) and MFI of the hypoxia probe (right, Hypoxia probe ${ }^{+} \mathrm{RBCs}$ ) in peripheral blood of Hbb ${ }^{\text {th } 3 /+}$ mice or WT mice, as detected by flow cytometry analysis. $n=10-13$ mice per group. Representative dot plots showing 1 out of 2 independent experiments. (B) Serum EPO was measured by ELISA. $n=10-13$. (C) Spleen Erfe (Fam132) ( $n=4-13$ mice per group) and liver Hamp (D) gene expression were measured by qPCR ( $n=$ 10-11 mice). (A-D) Individual values and mean \pm SD are shown. Statistical analysis was performed by comparing all treatment groups to the $\mathrm{Hbb}{ }^{\text {th } 3 /+}$ vehicle group using 1-way ANOVA with Dunnett's multiple comparison test. ${ }^{*} P<0.05 ;{ }^{* *} P<0.001$. 
A
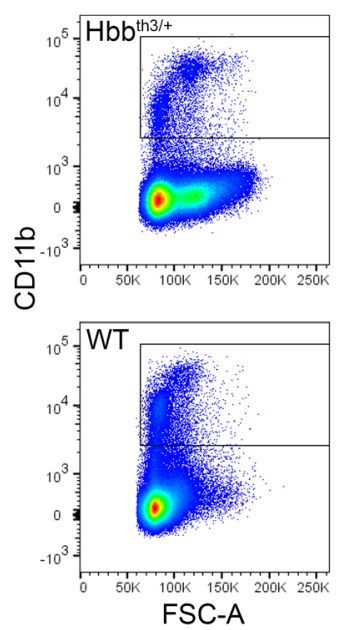
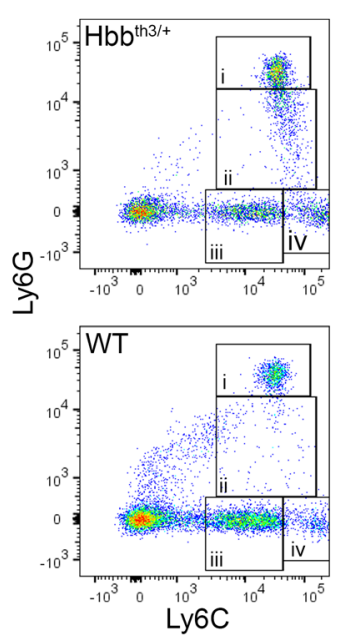

B

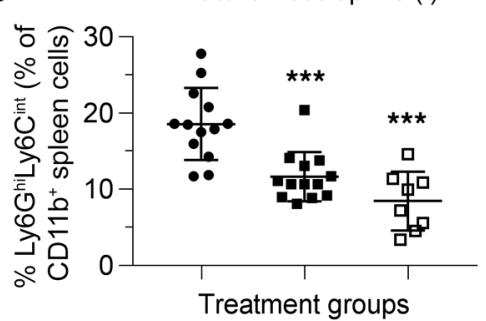

Resident monocytes (iii)

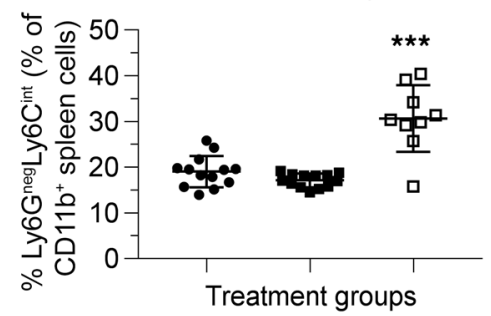

Immature myeloid cells (ii)

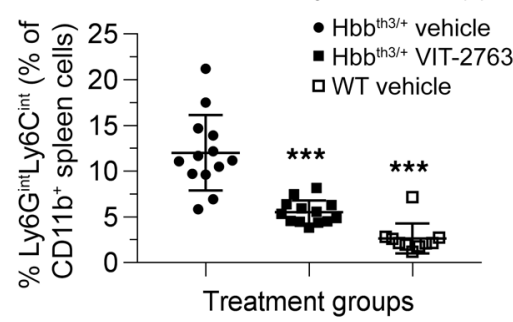

Inflammatory monocytes (iv)

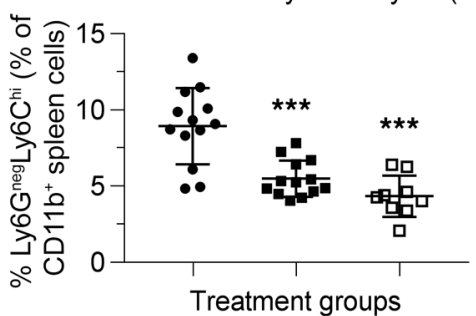

Figure 12. Effects of VIT-2763 on myeloid precursors in spleens of $\mathbf{H b b}^{\text {th } 3 /+}$ mice. (A) Gating strategy used to identify myeloid cell populations from the

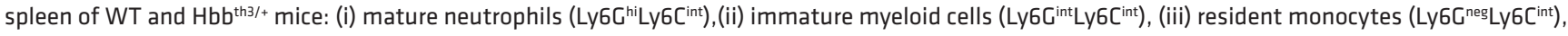
and (iv) inflammatory monocytes (Ly6G ${ }^{\text {nes }} L y 6 C^{\text {hi }}$ ). Representative dot plots from 1 out of 3 independent experiments are shown. (B) Quantification of percentages of mature neutrophils, immature myeloid cells, resident monocytes, and inflammatory monocytes in CD11 ${ }^{+}$spleen cells. Individual values and mean \pm SD are shown. $n=8-13$ mice per group. Statistical analysis was performed by comparing all treatment groups to the $\mathrm{Hbb}^{\text {th }} 3 /+$ vehicle group using 1-way ANOVA with Dunnett's multiple comparison test. ${ }^{* *} P<0.001$.

increase the production of endogenous hepcidin (siRNA or antisense DNA-targeting Tmprss6) are injectable drugs with higher molecular weight and no oral bioavailability. PK modeling using monkey plasma revealed that hepcidin has unfavorable pharmacological properties $\left(\mathrm{t}_{1 / 2}<2.5\right.$ minutes) (36) and, because of its 4 disulfide bonds, is particularly difficult to synthesize. Activin receptor ligand traps, such as sotatercept and luspatercept, are clinical-stage biologics that modify late-stage erythrocyte precursor cell differentiation and maturation (37). The longer halflife of these parenteral biologics offers the advantage of less frequent administration (once every 3 weeks) compared with an oral drug. However, the extended life span might become a disadvantage in cases of potential safety concerns. Indeed, the molecular target or targets of activin receptor traps are not well characterized, and reducing the activity of both known activin receptor ligands, GDF8 and GDF11, has been associated with increased risk of cardiovascular events (38). Therefore, VIT-2763 is anticipated to be what we believe is the first oral ferroportin inhibitor in clinical development with a well-defined target profile and offering important advantages of small molecules over parenteral medicines.

Ferroportin inhibitors are a class of structurally related small molecules that have been identified and optimized based on their potency to inhibit binding and internalization of fluorescent hepcidin (TMR-hepcidin) in J774 cell-expressing endogenous ferroportin. In addition, the internalization assay using MDCK cells expressing human ferroportin revealed that VIT-2763 induced ferroportin internalization similarly to hepcidin, although with slower kinetics and smaller maximal response. Interestingly, the initial hits identified in the library by the TMR-hepcidin ferroportin binding assay in J774 cells essentially lacked ferroportin internal- ization activity in MDCK ferroportin internalization assay (data not shown), suggesting that the potency of compounds above a certain threshold in ferroportin-binding assay is necessary for detectable ferroportin internalization activity and confirming the appropriate choice of the screening strategy depicted in Figure 1A.

VIT-2763 showed similar or higher potency in binding and iron efflux assays compared with hepcidin; however, the compound had about a 10-fold lower potency in the ferroportin internalization assay in MDCK cells. One possibility is that VIT-2763 directly blocks iron export by occluding ferroportin, which is similar to what has been shown for hepcidin (13). The incomplete ferroportin degradation observed in MDCK cells even after 18 hours exposure to VIT-2763 might be suggestive of the occlusion mode of action. Cells expressing endocytosis-deficient ferroportin or RBCs, which lack endocytosis machinery, will be helpful to experimentally address the ability of VIT-2763 to occlude ferroportin. Interestingly, hepcidin triggered formation of detectable intracellular vesicles in MDCK cells, which were less pronounced in VIT-2763-treated cells. The different pattern of the intracellular vesicles in MDCK cells triggered by VIT-2763 might reflect distinct pathways of ferroportin degradation.

Immunoprecipitation studies in $\mathrm{J} 774$ cells showed that the small molecule VIT-2763 also triggered rapid ubiquitination of endogenous ferroportin, as previously shown for hepcidin (24). It remains to be studied whether the different degree of ubiquitination of ferroportin induced by hepcidin and VIT-2763 targets ferroportin to different endocytic pathways. We have investigated the effect of VIT-2763 in duodenum, spleen, and liver by Western blot and quantitative PCR (qPCR) from $\mathrm{Hbb}^{\mathrm{th} 3 /+}$ and WT mice. VIT2763 did not reduce ferroportin levels in these tissues, as previously shown for minihepcidins (13), despite substantially decreasing 
serum iron (data not shown). The absence of ferroportin degradation, together with the reduction of serum iron by VIT-2763, is consistent with the mechanism of ferroportin occlusion. The reasons for the differential effect of hepcidin agonists on ferroportin endocytosis in cells and tissues are not clear. One possibility, discussed by Aschemeyer et al. (13), is different glycosylation of ferroportin and/or ferroportin-interacting proteins in cell lines versus tissues, which may differentially affect agonist binding to ferroportin and endocytosis pathways. Alternatively, the lack of degradation of ferroportin in spleen lysates could be due to analysis of the total membrane fraction (including plasma membrane and endosomes) by Western blot.

VIT-2763 is systemically available and most likely blocks iron export in all ferroportin-expressing tissues, including duodenum, spleen, and liver. Recent publications showed that ferroportin is highly abundant in mature RBCs and contributes a significant amount of iron to the blood $(10,11)$. It is therefore plausible that erythroid ferroportin inhibition by VIT-2763 contributes to the fast reduction of systemic iron observed in rodents. We are currently addressing the effect of VIT-2763 on RBCs.

Our data showed VIT-2763 ameliorated anemia, improved erythropoiesis, and decreased organ iron loading in the $\mathrm{Hbb}^{\text {th } 3 /+}$ $\beta$-thalassemia intermedia disease model. Presumably, iron restriction by VIT-2763 slows down the formation of $\mathrm{Hb}$ in developing erythroid cells, leading to reduction of toxic $\alpha$-globin aggregates in erythrocytes and associated oxidative stress. Similarly, induction of endogenous hepcidin by Tmprss6-targeting oligonucleotides or injection of hepcidin mimetics, such as minihepcidin in the $\mathrm{Hbb}^{\text {th } 3 /+}$ model resulted in decreased $\alpha$-globin aggregates in membranes of RBCs (18-20). Hb assembly is assisted by the molecular chaperone $\alpha$-Hb-stabilizing protein (AHSP), which specifically binds to free $\alpha$-globin chains before they associate with $\beta$-globin chains and prevents their aggregation (39). However in $\beta$-thalassemia, excess $\alpha$-globin chains exceed the capacity of AHSP and the unstable free $\alpha$-globin molecules undergo autooxidation and generate ROS that initiates a cascade of events leading to hemolysis and ineffective erythropoiesis (40). In addition, genetic evidence in patients supports the idea that reduction in $\alpha$-globin chain, due to coinherited $\alpha$-thalassemia, is beneficial in patients with $\beta$-thalassemia (3). The molecular mechanism of how iron restriction by ferroportin inhibition improves symptoms in $\beta$-thalassemia disease models needs further investigation.

VIT-2763 dose dependently decreased $\mathrm{MCH}$, as shown previously for hepcidin and hepcidin agonists in the $\mathrm{Hbb}^{\text {th } 3 /+}$ model $(20,27)$. These data indicate that iron restriction by VIT-2763 is reducing the erythroid iron intake per cell, resulting in lower $\mathrm{MCH}$, but increasing the number of RBCs, thereby improving the anemia (total $\mathrm{Hb}$ is increased). Overall, the decrease in $\mathrm{MCH}$ and MCV is associated with beneficial effects in the thalassemia model. Therefore, dose-finding clinical studies with VIT-2763 will allow avoidance of excessive iron restriction and preserve a stable number of RBCs by tightly monitoring hematological indices, such as MCH, MCV, and RBC counts. The moderate half-life of VIT-2763 in plasma and the high degree of flexibility on dosing provide important advantages in finely tuning the dose for optimal iron-restriction effect.
One important consequence of iron restriction by VIT-2763 is the decreased formation of ROS in developing erythroblasts in BM and spleen as well as in RBCs of $\mathrm{Hbb}^{\text {th } 3 /+}$ mice. Interestingly, $10 \%-$ $15 \%$ of mature RBCs in $\mathrm{Hbb}^{\text {th } 3 /+}$ mice have mitochondria, which are absent in WT RBCs. Similar abnormal retention of mitochondria in mature RBCs has been reported in patients and mice with sickle cell disease (41). Moreover, RBCs with mitochondria produced excessive levels of ROS. In healthy individuals, mitochondria disappear from RBCs during development as an adaptation to avoid generation of ROS (42). Notably, RBCs of $\mathrm{Hbb}^{\text {th } 3 /+}$ mice receiving VIT-2763 markedly reduced the proportion of mature RBCs containing mitochondria and producing elevated ROS, indicating improved terminal differentiation. To our knowledge, the effect of VIT-2763 on mitochondria in the $\mathrm{Hbb}^{\text {th } 3 /+}$ model has not been reported for other drugs targeting $\beta$-thalassemia.

VIT-2763 normalized myeloid spleen cell composition, most likely as a consequence of the corrected extramedullary erythropoiesis by VIT-2763. The ability of VIT-2763 to correct the proportion of spleen myeloid cells in the $\mathrm{Hbb}^{\mathrm{th} 3 /+}$ model indicates a potential for decreasing inflammation in $\beta$-thalassemia. This effect has not been reported for other drugs targeting $\beta$-thalassemia.

Patients with non-transfusion-dependent $\beta$-thalassemia (NTDT) have a moderate anemia, but still develop iron overload due to ineffective erythropoiesis and inadequate hepcidin production. The most severe form of $\beta$-thalassemia, transfusion-dependent $\beta$-thalassemia (TDT), requires regular blood transfusions (more than 5 $\mathrm{RBC}$ transfusions in 24 weeks), leading to secondary iron overload requiring iron chelation therapy (43). Blood transfusions and iron chelation therapy do not address the underlying pathological mechanism of the disease and are associated with increased risk of infection and adverse reactions.

The remarkable efficacy of VIT-2763 in $\mathrm{Hbb}^{\text {th } 3 /+}$ mice and the good tolerability profile in the nonclinical safety studies provide a direct rationale for investigating the efficacy of VIT-2763 in NTDT. VIT-2763 has not yet been tested in a mouse model of TDT. However, based on the mode of action of the compound, beneficial effects may be expected. Patients with TDT have severe iron overload due to regular blood transfusions. Blood transfusion causes a transient upregulation of hepcidin, which returns to basal values when the $\mathrm{Hb}$ levels decrease (44). Prevention of intestinal iron absorption by VIT-2763 during the intervals between transfusions might help to reduce further iron loading in TDT patients. More importantly, blood transfusion generates nontransferrin bound iron (NTBI), which is released by macrophages recycling damaged RBCs and triggers oxidative stress and vascular damage (45). Moreover, thalassemia patients on regular blood transfusions and chelation had elevated NTBI levels that correlated to the presence of heart disease (46). The oral ferroportin inhibitor VIT-2763 might prevent these noxious effects by sequestrating iron in macrophages and therefore interrupting a vicious cycle in $\beta$-thalassemia. Scavenging NTBI by apo-transferrin treatment was found to attenuate transfusionmediated increases in plasma NTBI and associated excess tissue iron loading $(47,48)$. Nevertheless, testing the efficacy of VIT-2763 in a TDT disease model will help to address this hypothesis.

In summary, by inhibiting ferroportin and thereby limiting the availability of iron to erythroid precursors, VIT-2763 decreases the formation of $\alpha$-globin aggregates generating ROS and 
improves the efficiency of erythropoiesis. In turn, the resulting $\mathrm{RBCs}$ with increased $\mathrm{Hb}$ supply more oxygen to tissues, which suppresses the hypoxia response and normalizes iron absorption. The clinical development of the oral ferroportin inhibitor has the potential to provide a therapeutic option with dosing convenience for patients with $\beta$-thalassemia and other diseases with dysregulated iron homeostasis.

\section{Methods}

TMR-hepcidin internalization assay in J774 cells. J774 cells (DSMZ) harvested from $80 \%$ confluent cultures were plated at $8 \times 10^{5}$ cells/ $\mathrm{ml}$ in complete DMEM medium supplemented with FBS, penicillinstreptomycin (Gibco, Thermo Fisher Scientific), $200 \mu \mathrm{M}$ Fe(III)-NTA (Fe[III\}-chloride and Nitrilotriacetic acid, Sigma-Aldrich), in 96-well MicroClear plates (Greiner) and grown at $37^{\circ} \mathrm{C}$. After overnight incubation, cells were washed and serial dilutions of test compounds were added in triplicate. $\mathrm{J774}$ cells were preincubated with compounds for 15 minutes before addition of TMR-hepcidin (provided by Sukhi Bansal, King's College London, London, United Kingdom) at $25 \mathrm{nM}$. Cells were incubated for 2 hours, and Hoechst 33342 dye (Thermo Fisher Scientific) was added to a final concentration of $0.5 \mu \mathrm{g} / \mathrm{ml}$. Cells were washed with Dulbecco's PBS (DPBS, Gibco, Thermo Fisher Scientific) and fixed using 5.3\% paraformaldehyde (Electron Microscopy Sciences) for 15 minutes. TMR (530-550 nm excitation/575-625 nm emission/250 ms exposure time) and Hoechst 33342 fluorescence images were acquired using a ScanR Plate Imager (Olympus) with a $\times 20$ high NA objective. Four pictures were acquired per well and fluorescence channel covering 1500 cells/well. The acquired data were analyzed with ScanR image analysis software. Image analysis included detection of nuclei, identification of cell-associated regions, application of a virtual channel, and thresholding for rolling-ball-type background reduction, followed by application of the sum(mean) algorithm to measure the TMR fluorescence associated with cells as a quantitative measure for internalized TMR-hepcidin.

Fluorescence polarization assay with recombinant human ferroportin and TMR-hepcidin. A mixture of $1.95 \mu \mathrm{M}$ human recombinant ferroportin isolated from Pichia pastoris yeast cells expressing human ferroportin with a C-terminal FLAG affinity tag (49) and $30 \mathrm{nM}$ TMRhepcidin in FP assay buffer containing $50 \mathrm{mM}$ Tris- $\mathrm{HCl}$ pH 7.3, 200 $\mathrm{mM} \mathrm{NaCl}$ (Sigma-Aldrich), 0.02\% $n$-dodecyl- $\beta$-D-maltopyranoside (DDM) (D310S, Sol-Grade 98\% pure, Anatrace), 0.1\% BSA (Sigma Aldrich), was plated into a 384-well black low-volume round-bottom plate (Corning) at $16 \mu \mathrm{l}$ per well. A volume of $8 \mu \mathrm{l}$ of serial dilutions of test compounds were added in duplicates to reach final ferroportin and TMR-hepcidin concentrations of $0.65 \mu \mathrm{M}$ and $10 \mathrm{nM}$, respectively. Plates were incubated for 90 minutes at room temperature and parallel (Fpara) and perpendicular (Fperp) fluorescence was measured in a Synergy H1 fluorescence reader (BioTek). FP values were calculated in $\mathrm{mP}$ according to the following formula: $\mathrm{mP}=(($ Fpara - Fperp $) /($ Fpara + Fperp)) $\times 1000$.

Iron efflux assay in T47D cells. Human T47D (ECACC) epithelial breast cancer cells were plated in 24-well plates (Greiner) at 350,000 cells/well and incubated overnight with $100 \mu \mathrm{M}^{58} \mathrm{Fe}$ (II) sulfate (Vifor [International] Ltd.) in $500 \mu \mathrm{M}$ L-ascorbic acid (Sigma Aldrich) containing DMEM growth medium. T47D cells were washed once with $500 \mu$ iron uptake buffer (IUB) (20 mM PIPES, $5 \mathrm{mM}$ glucose monohydrate, $\left.130 \mathrm{mM} \mathrm{NaCl}, 10 \mathrm{mM} \mathrm{KCl}, 1 \mathrm{mM} \mathrm{MgSO}{ }_{4}\right)$, then once with iron removal buffer (100 $\mu \mathrm{M}$ bathophenanthrolinedisulfonic acid disodium salt trihydrate and $500 \mu \mathrm{M} \mathrm{Na}_{2} \mathrm{~S}_{2} \mathrm{O}_{4}$ in IUB, Sigma-Aldrich) and again twice with IUB. Serial dilutions of hepcidin or VIT-2763 were added to each well in a total volume of $0.6 \mathrm{ml}$. Cells were incubated in growth medium without FBS at $37^{\circ} \mathrm{C}$ for 20 hours. ${ }^{58} \mathrm{Fe}$ was measured using inductively coupled plasma mass spectrometry (ICPMS) (Thermo Scientific, Element 2). Results are plotted as ng ${ }^{58} \mathrm{Fe}$ in supernatant per mg protein in cell lysates.

Ferritin-BLA reporter assay in HEK-FPN1-GFP cells. The HEKFPN1-GFP ferritin-BLA cell clone was generated by stable integration of (a) a human FPN1-GFP fusion construct inserted into a derivative of the doxycycline-inducible pTRE-Tight-BI plasmid (Clontech) and (b) a human ferritin promoter-BLA reporter gene into a derivative of the HEK-293 Tet-ON Advanced cell line (Clontech). To generate the ferritin-BLA reporter gene construct, a $1.4 \mathrm{~kb}$ fragment of the human ferritin $\mathrm{H}$ promoter was amplified by PCR from human genomic DNA (forward primer, 5'-CAGGTTTGTGAGCATCCTGAA-3'; reverse primer, 5'-GGCGGCGACTAAGGAGAGG-3') and inserted in front of the BLA gene present in the pcDNA 6.2/cGHFP-BLAzer-DEST plasmid (Thermo Fisher), thereby replacing the original CMV promoter and placing the iron response element (IRE) that regulates translation of the ferritin gene ca. 170 bp upstream of the start codon of the BLA reporter gene. HEK-BLA cells were seeded at $1.8 \times 10^{5}$ cells $/ \mathrm{ml}$ in DMEM/F12 containing 10\% FBS (Clontech), 1\% penicillin-streptomycin, $200 \mu \mathrm{g} / \mathrm{ml}$ hygromycin B, $5 \mu \mathrm{g} / \mathrm{ml}$, blasticidin (Thermo Fisher Scientific), and $4 \mu \mathrm{g} / \mathrm{ml}$ doxycycline (Clontech) in 384-well poly-Dlysine-coated microplates. Dilution series of the test compound was added in quadruplicate, and plates were incubated overnight at $37^{\circ} \mathrm{C}$. Cells were washed 3 times with HBSS. BLA activity was detected by adding the GeneBlazer reagent CCF4-AM (Thermo Fisher Scientific) to the cells. After incubation of the plates at $18^{\circ} \mathrm{C}$ for 60 minutes in darkness, blue and green fluorescence signals were measured in a Safire2 fluorescence plate reader (Tecan) with excitation at $410 \mathrm{~nm}$ and emissions at $458 \mathrm{~nm}$ (blue) and $522 \mathrm{~nm}$ (green). The ratio of blue $(458 \mathrm{~nm})$ and green $(522 \mathrm{~nm})$ fluorescence as a measure for BLA activity was calculated and $\mathrm{EC}_{50}$ values were determined with the calculated blue/ green fluorescence ratios.

FPN1-HaloTag-TMR internalization assay in MDCK cells. The assay quantifies ferroportin internalization triggered by hepcidin or VIT-2763 through microscopic detection of the disappearance of cell surface-associated fluorescently labeled ferroportin in a MDCK cell clone. The MDCK cell clone stably expresses human ferroportin with a C-terminal HaloTag fusion (Promega) and could be covalently labeled with HaloTag TMR-ligand. Cells were plated at $3 \times 10^{5}$ cells/ $\mathrm{ml}$ in DMEM medium and grown at $37^{\circ} \mathrm{C}$ overnight. MDCK cells were stained with $2 \mu \mathrm{M}$ HaloTag TMR-ligand (Promega) in growth medium for 20 minutes at $37^{\circ} \mathrm{C}, 5 \% \mathrm{CO}_{2}$ in the dark. Cells were then treated with serial dilutions of VIT-2763 or human hepcidin (Bachem) and incubated overnight at $37^{\circ} \mathrm{C}$. Nuclei were counterstained with $3 \mu \mathrm{M}$ Draq5 (eBioscience), and cells were washed twice with DPBS $+\mathrm{CaCl}_{2}$ $+\mathrm{MgCl}_{2}$ (BioTek 405 washer) and subsequently fixed with $4 \%$ paraformaldehyde in DPBS for 15 minutes. After 3 DPBS washes of cells, fluorescence images were acquired using an inverted IX81 epifluorescence microscope (ScanR, Olympus) and a $\times 20$ high NA objective. Four pictures were acquired per well for each channel (TMR and CY5), and the acquired data were analyzed using the Columbus image data storage and analysis system (PerkinElmer). Texture analysis (SER- 
Ridge) was used to quantify the disappearance of surface ferroportin triggered by hepcidin or VIT-2763, measured by a decrease of edge structures in the TMR channel.

Immunoprecipitation and Western blot. J774 cells were seeded at $12 \times 10^{6}$ cells per Petri dish in complete DMEM medium containing $200 \mu \mathrm{M} \mathrm{Fe}$ (III)-NTA and grown overnight at $37^{\circ} \mathrm{C}$. Cells were incubated with human hepcidin $(150 \mathrm{nM})$ or VIT-2763 (100 nM) for 10 , $20,40,60$, or 120 minutes. Cells were washed and lysed with ice-cold IP lysis buffer (Pierce, Thermo Scientific), including $1 \times$ HALT protease inhibitor cocktail (Thermo Scientific) and $10 \mathrm{mM}$ iodoacetamide (Sigma Aldrich) to stabilize ubiquitinated proteins. IP was done using the Pierce Classic IP Kit (Thermo Scientific) following the manufacturer's protocol. Cell lysate was incubated with the affinity purified rabbit anti-ferroportin antibody MTP-1 (catalog MTP11-A, Alpha Diagnostic International).

After IP, samples were analyzed by Western blotting using the affinity purified rabbit anti-mouse F308 antibody raised against a GST fusion protein of mouse ferroportin aa 224-308 (Vifor [International] Ltd.) and a mouse anti-mono- and polyubiquitinylated conjugates monoclonal antibody (Enzo Life Sciences, catalog BML-PW8810) for detection of ferroportin and ubiquitin, respectively. Mouse monoclonal anti-rabbit IgG light chain (Abcam, catalog ab99697) and antimouse IgG H\&L (Abcam, catalog ab6789) HRP conjugates were used as secondary antibodies.

Mouse strains and housing. C57BL/6 mice were purchased from Charles River. $\mathrm{Hbb}^{\text {th } 3 /+}$ mice (C57BL/6 background) were purchased from Jackson Laboratory (stock 002683) (50) and bred under specific pathogen-free conditions in the animal facility of Vifor (International) Ltd. The facility employs a 12-hour dark/12-hour light cycle, and all mice were provided water and food ad libitum. Mice were group- housed and maintained on standard rodent diet (Granovit, $250 \mathrm{ppm} \mathrm{mg} / \mathrm{kg}$ iron content) unless otherwise stated. Individual cages were randomly assigned to the treatment groups for equal distribution of sex and ages.

Efficacy of VIT-2763 in $\mathrm{Hbb}^{\text {th3/+ }}$ mice. Eight- to twelve-week-old male and female $\mathrm{Hbb}^{\text {th } 3 /+}$ mice ( $n=8$ to 13 ) were treated with either vehicle (0.5\% methylcellulose) or VIT-2763 at the indicated doses by oral gavage twice daily for the specified duration. The dosing was performed in the dark phase in the facility room, corresponding to the active period of rodents. WT littermates dosed with vehicle served as controls. To distinguish iron preloaded in mice at study start from iron acquired during the study period, mice were fed a low-iron diet (Granovit, $<10 \mathrm{mg} / \mathrm{kg}$ iron content) during the study period and the stable iron isotope ${ }^{58} \mathrm{Fe}$ was supplemented into the drinking water (1 $\mathrm{mM}^{58} \mathrm{FeSO}_{4}$ with $10 \mathrm{mM}$ ascorbic acid as a reducing agent) during the 6-hour period between both doses of compound. For the remaining 18 hours, all animals had access to mineral water without iron. Hb levels were determined weekly in tail-vein blood using a Hemocue Hb 201 device. Animals were sacrificed 3 hours after the final dose.

Flow cytometry for ROS, erythropoiesis, myelopoiesis, mitochondria, hypoxia, and PS exposure. Erythroid cells from BM and spleen were analyzed by flow cytometry. Distinct stages of RBC precursors were identified based on expression levels of Ter119 (APC-conjugated rat anti-mouse Ter119, eBioscience, catalog 17-5921), CD44 (APC-Cy7conjugated rat anti-mouse CD44, BioLegend, catalog 103028), CD71 (PE-conjugated rat anti-mouse CD71, eBioscience, catalog 12-0711), and the forward scatter (FSC) as a cell size measure (51). ROS in mature
RBCs were detected with the indicator CM-H2DCFDA (Invitrogen) after gating on Ter119 ${ }^{+}$and CD71- cells. Myelopoiesis was assessed in single spleen cell suspension. Mature neutrophils were identified as $C D 11 b^{+}$Ly6 $\mathrm{G}^{\text {hi }} \mathrm{Ly} 6 \mathrm{C}^{\text {int }}$, immature myeloid cells were identified as CD11b ${ }^{+}$Ly $6 G^{\text {int }} L y 6 C^{\text {int }}$, inflammatory monocytes were identified as

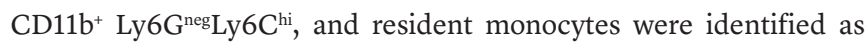
CD11b ${ }^{+}{\text {Ly } 6 G^{\text {neg }}}^{\text {Ly } 6 C^{\text {int }}}$ using FITC-conjugated rat anti-mouse CD11b (catalog 11-0112-82), PE-conjugated rat anti-mouse Ly6G (catalog 12-9668-82), and APC-conjugated rat anti-mouse Ly6C (catalog 17-5932-82) all from eBioscience. Mitochondria were detected using MitoTracker Deep Red FM (Invitrogen) in RBCs labeled with Ter119 and CD71 antibodies in a combination with CM-H2DCFDA staining for ROS detection.

Intracellular hypoxia was measured using the ROS-ID Hypoxia/ Oxidative Stress Detection Kit (Enzo Life Sciences, ENZ-51042K500). RBCs were labeled with Ter119 antibody and incubated with the hypoxia detection probe according to the manufacturer's instructions. PS exposure was detected using the Annexin V Apoptosis Detection Kit (Invitrogen) on peripheral blood cells labeled with Ter119 and CD71 antibodies.

Statistics. For quantification of $\mathrm{EC}_{50}$ or $\mathrm{IC}_{50}$ in the assays, for each data set, the fit of the log(inhibitor) vs. response (3 parameters) model was compared with the fit of the log(inhibitor) vs. response - variable slope (4 parameters) model and the data of the preferred model were used. Statistical analysis for parameters over time course was performed using a 2-way ANOVA with repeated measures for time-course effects. Where significant effects were observed, post tests were performed using Dunnett's multiple comparison test. For analysis of endpoint parameters, 1-way ANOVA with Dunnett's multiple comparison test was used. Data are presented by individual value with mean as scatter plots. Significant differences between treatment groups compared with $\mathrm{Hbb}^{\text {th } 3 /+}$ vehicle group are indicated. Statistical analyses and $\mathrm{EC}_{50} / \mathrm{IC}_{50}$ value calculations were carried out with Prism software (GraphPad Prism version 7.04).

See Supplemental Methods for additional information.

Study approval. The animal studies described in this paper complied with all applicable sections of the law and associated guidelines and were approved by the Veterinary Department of Zurich. All studies were also performed in compliance with the Code of Conduct of Vifor Pharma Group.

\section{Author contributions}

VM wrote the manuscript with input from NN, HS, and FD. VM and FD provided guidance in designing of hypothesis and experiments. NN and HS planned experiments and analyzed data. AF, PA, AV, and CD performed experiments and analyzed data. FD was responsible for project conception.

\section{Acknowledgments}

We thank the following colleagues from Vifor Pharma Group for their invaluable contributions: Stefan Reim and the chemical development team for upscaling of VIT-2763; Anna-Lena Steck, Jörg Schmitt, and Maria Wilhelm from the analytical development team for ICP-MS and ICP-OES analysis of tissue iron and stability tests of VIT-2763; Erik Philipp and Roland Riederer for providing ${ }^{58} \mathrm{Fe}$ (II) $\mathrm{SO}_{4}$; Claudio Mori and Chris Rapier for manuscript review; and Bjarte Furnes for overseeing the nonclini- 
cal safety program. We thank the following collaborators from Evotec SE that contributed to this project: Chris Yarnold and the Discovery chemistry team; and Marc Slack and the Cellular Assays team. Synthetic TMR-hepcidin was kindly provided by Sukhi Bansal, King's College London. The recombinant human ferroportin produced in yeast was kindly provided by Maria Carmela Bonaccorsi and Gianni Musci from University of Rome La Sapienza and University of Molise, respectively. René Prétôt and Hugo Albrecht of University of Applied Sciences and Arts Northwestern Switzerland and University of South Australia, respectively, are kindly acknowledged for generating the ferritin reporter cell line with inducible ferroportin expression. Special thanks are given to the animal facility head Marco Franchini and animal caretaker Martin Haenggi. We thank Giovanni Pellegrini and the team of LAMP, University of Zurich, for being involved and helpful with histological studies.

Address correspondence to: Vania Manolova, Rechenstrasse 37, St. Gallen, Switzerland; Phone: 41.58.851.81.72; Email: vania. manolova@viforpharma.com.

AV's present address is: Roche Glycart AG, Schlieren, Switzerland.
1. Rivella S. Iron metabolism under conditions of ineffective erythropoiesis in $\beta$-thalassemia. Blood. 2019;133(1):51-58.

2. Taher AT, Weatherall DJ, Cappellini MD. Thalassaemia. Lancet. 2018;391(10116):155-167.

3. Mettananda S, Gibbons RJ, Higgs DR. $\alpha$-Globin as a molecular target in the treatment of $\beta$-thalassemia. Blood. 2015;125(24):3694-3701.

4. Rivella S. $\beta$-thalassemias: paradigmatic diseases for scientific discoveries and development of innovative therapies. Haematologica. 2015;100(4):418-430.

5. Rivella S. The role of ineffective erythropoiesis in non-transfusion-dependent thalassemia. Blood Rev. 2012;26(Suppl 1):S12-S15.

6. Nemeth E, et al. Hepcidin regulates cellular iron efflux by binding to ferroportin and inducing its internalization. Science. 2004;306(5704):2090-2093.

7. Donovan A, et al. Positional cloning of zebrafish ferroportin1 identifies a conserved vertebrate iron exporter. Nature. 2000;403(6771):776-781.

8. McKie AT, et al. A novel duodenal iron-regulated transporter, IREG1, implicated in the basolateral transfer of iron to the circulation. Mol Cell. 2000;5(2):299-309.

9. Muckenthaler MU, Rivella S, Hentze MW, Galy B. A red carpet for iron metabolism. Cell. 2017;168(3):344-361.

10. Zhang DL, Ghosh MC, Ollivierre H, Li Y, Rouault TA. Ferroportin deficiency in erythroid cells causes serum iron deficiency and promotes hemolysis due to oxidative stress. Blood. 2018;132(19):2078-2087.

11. Zhang DL, et al. Erythrocytic ferroportin reduces intracellular iron accumulation, hemolysis, and malaria risk. Science. 2018;359(6383):1520-1523.

12. Rivera S, Nemeth E, Gabayan V, Lopez MA, Farshidi D, Ganz T. Synthetic hepcidin causes rapid dose-dependent hypoferremia and is concentrated in ferroportin-containing organs. Blood. 2005;106(6):2196-2199.

13. Aschemeyer S, et al. Structure-function analysis of ferroportin defines the binding site and an alternative mechanism of action of hepcidin. Blood. 2018;131(8):899-910.

14. Anderson SA, et al. The IRP1-HIF-2 $\alpha$ axis coordinates iron and oxygen sensing with erythropoiesis and iron absorption. Cell Metab. 2013;17(2):282-290.

15. Kattamis A, et al. The effects of erythropoetic activity and iron burden on hepcidin expression in patients with thalassemia major. Haematologi- ca. 2006;91(6):809-812.

16. Schwartz AJ, et al. Hepatic hepcidin/intestinal HIF- $2 \alpha$ axis maintains iron absorption during iron deficiency and overload. JClin Invest. 2019;129(1):336-348.

17. Kautz L, Jung G, Valore EV, Rivella S, Nemeth E, Ganz T. Identification of erythroferrone as an erythroid regulator of iron metabolism. Nat Genet. 2014;46(7):678-684.

18. Casu C, et al. Minihepcidin peptides as disease modifiers in mice affected by $\beta$-thalassemia and polycythemia vera. Blood. 2016;128(2):265-276.

19. Schmidt PJ, et al. An RNAi therapeutic targeting Tmprss6 decreases iron overload in Hfe(-/-) mice and ameliorates anemia and iron overload in murine $\beta$-thalassemia intermedia. Blood. 2013;121(7):1200-1208.

20. Guo S, et al. Reducing TMPRSS6 ameliorates hemochromatosis and $\beta$-thalassemia in mice. J Clin Invest. 2013;123(4):1531-1541.

21. Preza GC, et al. Minihepcidins are rationally designed small peptides that mimic hepcidin activity in mice and may be useful for the treatment of iron overload. J Clin Invest. 2011;121(12):4880-4888.

22. Richard F, van Lier JJ, Roubert B, Haboubi T, Gohring UM, Durrenberger F. Oral ferroportin inhibitor VIT-2763: First-in-human, phase 1 study in healthy volunteers [published online ahead of print October 31, 2019]. Am J Hematol. https://doi.org/10.1002/ajh.25670.

23. Nemeth E, Preza GC, Jung CL, Kaplan J, Waring AJ, Ganz T. The N-terminus of hepcidin is essential for its interaction with ferroportin: structure-function study. Blood. 2006;107(1):328-333

24. Qiao B, et al. Hepcidin-induced endocytosis of ferroportin is dependent on ferroportin ubiquitination. Cell Metab. 2012;15(6):918-924.

25. Ross SL, et al. Molecular mechanism of hepcidin-mediated ferroportin internalization requires ferroportin lysines, not tyrosines or JAK-STAT. Cell Metab. 2012;15(6):905-917.

26. Taniguchi R, et al. Outward- and inward-facing structures of a putative bacterial transition-metal transporter with homology to ferroportin. Nat Commun. 2015;6:8545.

27. Gardenghi S, et al. Hepcidin as a therapeutic tool to limit iron overload and improve anemia in $\beta$-thalassemic mice. J Clin Invest. 2010;120(12):4466-4477.

28. Voskou S, Aslan M, Fanis P, Phylactides M, Kleanthous M. Oxidative stress in $\beta$-thalassaemia and sickle cell disease. Redox Biol. 2015;6:226-239.
29. Sandoval H, et al. Essential role for Nix in autophagic maturation of erythroid cells. Nature. 2008;454(7201):232-235.

30. Khungwanmaythawee K, et al. Mitochondrial changes in $\beta^{\circ}$-thalassemia/Hb E disease. PLoS One. 2016;11(4):e0153831.

31. Leecharoenkiat $A$, et al. Increased oxidative metabolism is associated with erythroid precursor expansion in $\beta 0$-thalassaemia/Hb E disease. Blood Cells Mol Dis. 2011;47(3):143-157.

32. Kiefer CR, Snyder LM. Oxidation and erythrocyte senescence. Curr Opin Hematol. 2000;7(2):113-116

33. Li MO, Sarkisian MR, Mehal WZ, Rakic P, Flavell RA. Phosphatidylserine receptor is required for clearance of apoptotic cells. Science. 2003;302(5650):1560-1563.

34. Garcia-Santos D, et al. Inhibition of heme oxygenase ameliorates anemia and reduces iron overload in a $\beta$-thalassemia mouse model. Blood. 2018;131(2):236-246.

35. Siwaponanan P, et al. Reduced PU.1 expression underlies aberrant neutrophil maturation and function in $\beta$-thalassemia mice and patients. Blood. 2017;129(23):3087-3099.

36. Xiao JJ, et al. Pharmacokinetics of antihepcidin monoclonal antibody $\mathrm{Ab} 12 \mathrm{~B} 9 \mathrm{~m}$ and hepcidin in cynomolgus monkeys. AAPS J. 2010;12(4):646-657.

37. Suragani RN, et al. Modified activin receptor IIB ligand trap mitigates ineffective erythropoiesis and disease complications in murine $\beta$-thalassemia. Blood. 2014;123(25):3864-3872.

38. Olson KA, et al. Association of growth differentiation factor $11 / 8$, putative anti-ageing factor, with cardiovascular outcomes and overall mortality in humans: analysis of the Heart and Soul and HUNT3 cohorts. Eur Heart J. 2015;36(48):3426-3434.

39. Yu X, et al. An erythroid chaperone that facilitates folding of alpha-globin subunits for hemoglobin synthesis. JClin Invest. 2007;117(7):1856-1865.

40. Voon HP, Vadolas J. Controlling alpha-globin: a review of alpha-globin expression and its impact on beta-thalassemia. Haematologica. 2008;93(12):1868-1876.

41. Jagadeeswaran R, et al. Pharmacological inhibition of LSD1 and mTOR reduces mitochondrial retention and associated ROS levels in the red blood cells of sickle cell disease. Exp Hematol. 2017;50:46-52.

42. Moras M, Lefevre SD, Ostuni MA. From erythroblasts to mature red blood cells: organelle clear- 
ance in mammals. Front Physiol. 2017;8:1076.

43. Olivieri NF. The beta-thalassemias. NEngl J Med. 1999;341(2):99-109.

44. Pasricha SR, Frazer DM, Bowden DK, Anderson GJ. Transfusion suppresses erythropoiesis and increases hepcidin in adult patients with $\beta$-thalassemia major: a longitudinal study. Blood. 2013;122(1):124-133.

45. Baek JH, et al. Iron accelerates hemoglobin oxidation increasing mortality in vascular diseased guinea pigs following transfusion of stored blood.
JCI Insight. 2017;2(9):93577.

46. Piga A, et al. High nontransferrin bound iron levels and heart disease in thalassemia major. Am J Hematol. 2009;84(1):29-33.

47. Gelderman MP, et al. Reversal of hemochromatosis by apotransferrin in non-transfused and transfused Hbbth3/+ (heterozygous B1/ B2 globin gene deletion) mice. Haematologica. 2015;100(5):611-622.

48. Li H, et al. Transferrin therapy ameliorates disease in beta-thalassemic mice. Nat Med
2010;16(2):177-182.

49. Bonaccorsi di Patti MC, et al. A structural model of human ferroportin and of its iron binding site. FEBS J. 2014;281(12):2851-2860.

50. Yang B, Kirby S, Lewis J, Detloff PJ, Maeda N, Smithies O. A mouse model for $\beta^{\circ}$-thalassemia. Proc Natl Acad Sci USA. 1995;92(25):11608-11612.

51. Liu J, et al. Quantitative analysis of murine terminal erythroid differentiation in vivo: novel method to study normal and disordered erythropoiesis. Blood. 2013;121(8):e43-e49. 